Draft version August 7, 2018

Preprint typeset using $\mathrm{LATE}_{\mathrm{E}} \mathrm{X}$ style emulateapj v. 5/2/11

\title{
THE FASTEST GALAXY EVOLUTION IN AN UNBIASED COMPACT GROUP SAMPLE WITH WISE
}

\author{
Gwang-Ho Lee ${ }^{1}$, Ho Seong Hwang ${ }^{2}$, Jubee Sohn ${ }^{3}$, And Myung Gyoon Lee ${ }^{1}$ \\ ${ }^{1}$ Department of Physics and Astronomy, Seoul National University, 1 Gwanak-ro, Gwanak-gu, Seoul 08826, Republic of Korea \\ ${ }^{2}$ School of Physics, Korea Institute for Advanced Study, 85 Hoegiro, Dongdaemun-gu, Seoul 02455, Republic of Korea and \\ ${ }^{3}$ Smithsonian Astrophysical Observatory, 60 Garden Street, Cambridge, MA 02138, USA \\ Draft version August 7, 2018
}

\begin{abstract}
We study the mid-infrared (MIR) properties of galaxies in compact groups and their environmental dependence using the Wide-field Infrared Survey Explorer (WISE) data. We use a volume-limited sample of 670 compact groups and their 2175 member galaxies with $M_{r}<-19.77$ and $0.01<z<$ 0.0741, drawn from Sohn et al. (2016), which were identified using a friends-of-friends algorithm. Among the 2175 galaxies, 1541 galaxies are detected at WISE $12 \mu \mathrm{m}$ with a signal-to-noise ratio greater than 3. Among the 1541 galaxies, 433 AGN-host galaxies are identified by using both optical and MIR classification scheme. Using the remaining 1108 non-AGN galaxies, we find that the MIR [3.4] - [12] colors of compact group early-type galaxies are on average bluer than those of cluster early-type galaxies. When compact groups have both early- and late-type member galaxies, the MIR colors of the late-type members in those compact groups are bluer than the MIR colors of cluster latetype galaxies. As compact groups are located in denser regions, they tend to have larger early-type galaxy fractions and bluer MIR color galaxies. These trends are also seen for neighboring galaxies around compact groups. However, compact group member galaxies always have larger early-type galaxy fractions and bluer MIR colors than their neighboring galaxies. Our findings suggest that the properties of compact group galaxies depend on both internal and external environments of compact groups, and that galaxy evolution is faster in compact groups than in the central regions of clusters.

Subject headings: galaxies: evolution - galaxies: groups: general - galaxies: interactions
\end{abstract}

\section{INTRODUCTION}

Compact groups of galaxies contain several galaxies within just a few tens of kilo-parsec scales. This makes compact groups have extremely high galaxy number densities (the median galaxy number density of $\log \left(\rho /\left[h^{-3}\right.\right.$ $\left.\left.\mathrm{Mpc}^{3}\right]\right)=4.36$, Sohn et al. 2016), higher than the galaxy number densities of cluster and group environments (the median galaxy number density of $\log \left(\rho /\left[h^{-3}\right.\right.$ $\left.\left.\mathrm{Mpc}^{3}\right]\right)=1.97$ for clusters and groups in the A2199 supercluster, Lee et al. 2015; Sohn et al. 2015). The velocity dispersions of compact groups are much smaller $\left(<879 \mathrm{~km} \mathrm{~s}^{-1}\right.$ with a median value of $194 \mathrm{~km} \mathrm{~s}^{-1}$ with a standard deviation of $156 \mathrm{~km} \mathrm{~s}^{-1}$, Sohn et al. 2016. Table 7) than those of clusters $\left(\sim 400-1300 \mathrm{~km} \mathrm{~s}^{-1}\right.$ with a median value of $778 \mathrm{~km} \mathrm{~s}^{-1}$ and a standard deviation of $208 \mathrm{~km} \mathrm{~s}^{-1}$, Rines et al. 2013). These two characteristics make compact groups an ideal environment for frequent interactions and mergers between galaxies (e.g., Rubin et al. 1990; Rodrigue et al. 1995; Amram et al. 2007; Coziol \& Plauchu-Fravn 2007; Plauchu-Fravn \& Coziol 2010; Gallagher et al. 2010; Konstantopoulos et al. 2012; Sohn et al. 2013; Vogt et al. 2015).

Numerical simulations suggested that galaxies in a compact group should merge into a single elliptical galaxy within a few Gyrs, causing the compact group to disappear (Barnes 1985, 1989; Mamon 1987). Gallagher et al. (2010) also reached a similar conclusion through their study of star cluster age-dating and neutral hydrogen content in the Hickson compact group 31;

ghlee@astro.snu.ac.kr, mglee@astro.snu.ac.kr the compact group should merge into a single elliptical within 1 Gyr (see also Rubin et al. 1990). Kroupa (2015) suggested that the abundance of compact groups should decrease as the redshift decreases from $z \simeq 0.1$ to $z=0$ in the $\Lambda$ CDM universe. However, Sohn et al. (2015) found that the abundance of compact groups changes little with redshifts at $0.01<z<0.22$, using a spectroscopically complete sample of 332 compact groups. Kroupa (2015) also suggested an alternative scenario that compact groups do not merge, which predicts a constant abundance of compact groups at $z<0.1$. However, this alternative scenario is based on the assumption of a universe without dark matter.

Diaferio et al. (1994) suggested that compact groups replenish themselves with new members from the surrounding environment, thereby extending their lifetimes to the current epoch. The finding of Sohn et al. (2015), constant abundance of compact group at $0.01<$ $z<0.22$, can be explained by this replenishment model. This replenishment model is supported by several observational findings that showed that many ( $>50 \%$ ) compact groups are embedded in or associated with larger-scale structures (Rood \& Struble 1994; Ramella et al. 1994; de Carvalho et al. 1994; Ribeiro et al.|1998; Mendel et al.|2011; Pompei \& Iovino 2012). However, Díaz-Giménez \& Zandivarez (2015) showed that only $27 \%$ of compact groups are embedded in surrounding structures.

Unfortunately, many existing compact group catalogs suffer from some observational biases, which make them difficult to use for testing compact group formation models and for studying the relation between compact groups and their surrounding environments. For 
example, McConnachie et al. (2009) constructed a large sample of compact groups, from the photometric data $\left(m_{r}<21\right)$ of Sloan Digital Sky Survey (SDSS) Data Release 6 (DR6, Adelman-McCarthy et al. 2008), including 77,088 compact group candidates and their 313,508 tentative member galaxies. Their sample is the largest one currently, but it is highly contaminated with interlopers ( $>50 \%$, Mendel et al. 2011; Sohn et al. 2013) because the sample is based only on photometric information using Hickson's selection criteria (Hickson 1982). To reduce this contamination, Sohn et al. (2015) compiled the redshifts from Fred Lawrence Whipple Observatory (FLWO)/FAST observations and from the SDSS DR12 (Alam et al. 2015) for the photometric sample of compact group galaxies in McConnachie et al. (2009), and constructed a catalog of 332 compact groups. This catalog is currently the largest one with complete spectroscopic redshifts. However, this sample is affected by an isolation criterion from Hickson's selection criteria. The isolation criterion requires that no galaxies be present in a radius of up to three times the group radius. This criterion was adopted to avoid selecting very dense regions like cluster cores, but this could introduce a bias that misses nearby compact groups with apparently large sizes and embedded systems in dense environments (Barton et al. 1996).

Recently, Sohn et al. (2016) published a new catalog of 1588 compact groups and their 5178 member galaxies at $0.01<z<0.19$ using SDSS DR12 spectroscopic data supplemented by additional redshifts from the literature and from FLWO/FAST observations. They applied a friends-of-friends algorithm to identify the compact groups without using the isolation criterion. As a result, their catalog successfully contains nearby $(z<0.05)$ compact groups and embedded systems in dense regions. This is important in order to study the relation between compact groups and their surrounding environments without any sample bias.

Previous observational studies suggested that galaxy evolution in compact groups is faster than in the field from the comparisons of Lick indices (Proctor et al. 2004; Mendes de Oliveira et al. 2005; de la Rosa et al. 2007), the star formation quenching timescales (Plauchu-Frayn et al. 2012), specific star formation rates (SFRs) distribution (Tzanavaris et al. 2010; Coenda et al. 2015; Lenkić et al. 2016), and color distribution (Bitsakis et al. 2010, 2011, 2016; Walker et al. 2010, 2012). Proctor et al. (2004) and Mendes de Oliveira et al. (2005) found that the stellar ages and the early-type galaxy fractions of compact group galaxies are similar to those of cluster galaxies, which suggests that compact group galaxies evolve as fast as cluster galaxies.

On the other hand, Johnson et al. (2007) and Walker et al. (2010, 2012) showed that compact group galaxies exhibit a bimodal distribution in the Spitzer IRAC $(3.6-8.0 \mu \mathrm{m})$ color space with a statistically evident gap, so-called "canyon", between star-forming galaxies with MIR red colors and quiescent galaxies with MIR blue colors. Recently, Zucker et al. (2016) newly identified the canyon galaxies in the WISE colorcolor diagram. This gap may suggest the accelerated galaxy evolution in compact groups because the gap is not seen in comparison galaxy samples from field, from interacting pairs, and from the center of the Coma cluster (Walker et al. 2010, 2012, 2013). Cluver et al. (2013) found that compact group galaxies in the gap mostly show enhanced warm $\mathrm{H}_{2}$ emission from the observations with the Spitzer Infrared Spectrograph, which could be caused by shock heating (Appleton et al. 2006; Cluver et al. 2010). This result implies that shock heating may be responsible for rapid evolution of galaxies in compact groups.

However, these results are mainly based on the catalogs of compact groups selected using Hickson's criteria, which can introduce a sample bias. We therefore revisit the issues on the comparison of compact groups galaxies with cluster and field galaxies using the unbiased sample of compact groups from Sohn et al. (2016). In this paper, we use the Wide-field Infrared Survey Explorer (WISE, Wright et al. 2010) mid-infrared (MIR) data to study the environmental effects on compact group galaxies and the relation between compact groups and their surrounding environments. The MIR data are useful indicators of mean stellar ages (Piovan et al. 2003; Ko et al. 2009), especially for compact group galaxies that are mainly dominated by old stellar populations. Red-sequence galaxies with small amounts of young $(<1$ Gyr) and intermediate-age (1-10 Gyr) stellar populations can be distinguished from those without the populations in MIR color space (Ko et al. 2013, 2016; Lee et al. 2015). The sample we use in this paper contains 670 compact groups with 2175 member galaxies drawn from the catalog of Sohn et al. (2016). When we investigate the MIR properties of galaxies in Sections 3 and 4, we use 1541 member galaxies detected at $12 \mu \mathrm{m}$ with a signal-to-noise ratio $(\mathrm{S} / \mathrm{N})$ greater than 3 . This sample is larger than those of previous MIR studies. For example, the samples of Walker et al. (2012) and several previous studies (Johnson et al. 2007; Bitsakis et al. 2010, 2011; Walker et al. 2010) have less than 50 compact groups, the sample of Walker et al. (2013) has 99 compact groups and 348 member galaxies, and the sample of Zucker et al. (2016) has 163 compact groups and 567 member galaxies.

Section 2 describes the compact group sample and comparison samples of cluster/field galaxies. In Section 3 we compare the properties of galaxies in compact groups with those in other environments, and investigate how environment affects the properties of galaxies in compact groups. In Section 4 we discuss the environmental effects on galaxy evolution in compact groups and the relation between compact groups and their surrounding environments. In Section 5 we summarize our results and present conclusions. Throughout, we adopt flat $\Lambda \mathrm{CDM}$ cosmological parameters: $H_{0}=70 \mathrm{~km} \mathrm{~s}^{-1} \mathrm{Mpc}^{-1}, \Omega_{\Lambda}=0.7$, and $\Omega_{m}=0.3$.

\section{DATA}

Sohn et al. (2016) constructed a catalog of compact groups using the spectroscopic sample of SDSS DR12 galaxies with $m_{r}<17.77$. The completeness of the spectroscopic data in SDSS is low for bright galaxies at $m_{r}<14.5$ because of saturation and cross-talk in the spectrograph, and for the galaxies in high-density regions (e.g., galaxy clusters) because of the fiber collision (see Figure 1 of Park \& Hwang 2009). Therefore, Sohn et al. (2016) supplemented the catalog with redshifts from 
TABLE 1

SDSS-related Physical Parameters of Compact Group Galaxies

\begin{tabular}{cccccccc}
\hline \hline Host Group $^{a}$ & Galaxy ID & R.A. & Decl & Redshift & $M_{r}^{b}$ & $u-r^{c}$ Morph $^{d}$ \\
\hline V1CG001 & 1237648705657307198 & 198.233322 & 1.007515 & $0.073839 \pm 0.000029$ & $-19.82 \pm 2.21$ & $2.28 \pm 0.10$ & 2 \\
V1CG001 & 1237648705657307347 & 198.229294 & 1.010990 & $0.072679 \pm 0.000020$ & $-20.11 \pm 0.01$ & $2.57 \pm 0.06$ & 1 \\
V1CG001 & 1237648705657307315 & 198.218872 & 1.019821 & $0.070435 \pm 0.000017$ & $-21.16 \pm 0.01$ & $2.67 \pm 0.04$ & 1 \\
V1CG002 & 1237661126155436166 & 139.939529 & 33.745014 & $0.017118 \pm 0.000007$ & $-21.22 \pm 0.06$ & $2.77 \pm 0.01$ & 1 \\
V1CG002 & 1237661126155436169 & 139.922531 & 33.738174 & $0.020334 \pm 0.000013$ & $-20.72 \pm 0.05$ & $2.61 \pm 0.01$ & 2 \\
V1CG002 & 1237661126155436164 & 139.945221 & 33.749741 & $0.023006 \pm 0.000003$ & $-22.84 \pm 0.01$ & $2.91 \pm 0.01$ & 1 \\
V1CG003 & 1237661136886431890 & 154.733276 & 37.285831 & $0.048809 \pm 0.000019$ & $-20.41 \pm 0.01$ & $1.91 \pm 0.03$ & 2 \\
V1CG003 & 1237661136886497282 & 154.743576 & 37.300205 & $0.047580 \pm 0.000007$ & $-20.84 \pm 0.01$ & $1.68 \pm 0.01$ & 2 \\
V1CG003 & 1237661136886497353 & 154.747711 & 37.308155 & $0.047489 \pm 0.000008$ & $-20.08 \pm 0.01$ & $1.99 \pm 0.02$ & 2 \\
\hline
\end{tabular}

Note. - The full table is available in the online journal. A portion is shown here for guidance regarding its form and content. ${ }^{a}$ Group ID from Table 5 of Sohn et al. (2016).

${ }^{b}$ The $k_{z=0.1^{-}}$, and evolution-corrected $r$-band Petrosian absolute magnitudes.

${ }^{c}$ The SDSS extinction- and $k$-corrected model magnitudes.

${ }^{d}$ Galaxy morphology. 1 indicates an early-type galaxy, while 2 indicates a late-type galaxies.

the literature (Hill \& Oegerle 1993, 1998; Wegner et al. 1996, 1999; Slinglend et al. 1998; Falco et al. 1999; see also Hwang et al. 2010) and from FAST observations at FLWO (Sohn et al. 2015). They applied the friendsof-friends algorithm with a projected linking length of $\Delta D=50 h^{-1} \mathrm{kpc}$ and a radial linking length of $|\Delta V|=1000 \mathrm{~km} \mathrm{~s}^{-1}$, and constructed a magnitudelimited $\left(m_{r}<17.77\right)$ sample of 1588 compact groups with each consisting of three or more member galaxies at $0.01<z<0.19$. This new catalog contains 18 times as many systems and reaches three times the depth of the catalog of Barton et al. (1996), which is also based on the friends-of-friends algorithm.

Sohn et al. (2016) also constructed two volume-limited subsamples: the $\mathrm{V} 1$ sample of galaxies with $M_{r}<-19.77$ and $0.01<z<0.0741$ and the V2 sample of galaxies with $M_{r}<-20.77$ and $0.01<z<0.1154$ (V2) 1 . These volume-limited samples contain 670 and $297 \mathrm{com}-$ pact groups that are independently identified through the friends-of-friends algorithm. Unlike systems in the magnitude-limited sample, the volume-limited samples have systems with a median stellar mass independent of redshift. Therefore, the volume-limited samples allow us to study the properties of compact groups without any sample bias that could be introduced in the magnitudelimited sample. Details for the catalogs and the compact group selection are described in Sections 2 and 3 of Sohn et al. (2016).

In this study, we use the V1 sample of 670 compact groups and their 2175 member galaxies with $M_{r}<$ -19.77 and $0.01<z<0.0741$. To study the MIR properties of these galaxies, we cross-correlate the galaxies with the objects in the $A L L W I S E$ source catalog 2 using a matching tolerance of $3^{\prime \prime}$, corresponding to about half of the FWHM of the PSF at $3.4 \mu \mathrm{m}$. Among the 2175 galaxies, 2067 (95\%) are matched with ALLWISE sources. We use the profile-fitting magnitudes of the sources at four MIR bands $(3.4 \mu \mathrm{m}, 4.6 \mu \mathrm{m}, 12 \mu \mathrm{m}$, and $22 \mu \mathrm{m})$. When we investigate the MIR properties of galaxies, we use

1 Sohn et al. (2016) used $M_{r}<-19+5 \log h$ and $M_{r}<-20+$ $5 \log h$ with $h=1$ (i.e., $H_{0}=100 \mathrm{~km} \mathrm{~s}^{-1} \mathrm{Mpc}^{-1}$ ). However, we adopt $h=0.7$ (i.e., $H_{0}=70 \mathrm{~km} \mathrm{~s}^{-1} \mathrm{Mpc}^{-1}$ ), which results in $-19+5 \log h \simeq-19.77$ and $-20+5 \log h \simeq-20.77$.

${ }^{2}$ http://wise2.ipac.caltech.edu/docs/release/allwise/ only galaxies detected at $12 \mu \mathrm{m}$ with $\mathrm{S} / \mathrm{N}>3$. Because the ALLWISE magnitudes with $\mathrm{S} / \mathrm{N}<2$ are just upper limits, we choose only the galaxies with $\mathrm{S} / \mathrm{N}>3$ to safely use the magnitudes. Among the 2175 galaxies, 1541 galaxies $(71 \%)$ are detected at $12 \mu \mathrm{m}$. If we adopt the $\mathrm{S} / \mathrm{N}$ cut of 5 , the sample size is reduced from 1541 to 863 galaxies by $44 \%$. However, we confirm that our conclusions do not change much even if we use this small sample.

To compare the physical properties of compact group galaxies with those in other environments, we use two comparison samples of cluster and field galaxies. Hwang et al. (2012a) constructed a sample of 129 relaxed Abell clusters at $0.02<z<0.14$ using the spectroscopic sample of the SDSS DR7 Abazajian et al. 2009). The cluster sample also includes the galaxies at $R<10 R_{200}\left(R_{200}\right.$ is the virial radius of the cluster) and $\Delta V=\left|V_{\text {gal }}-V_{\mathrm{cl}}\right|<1000 \mathrm{~km} \mathrm{~s}^{-1}\left(V_{\text {gal }}\right.$ and $V_{\mathrm{cl}}$ are radial velocity of a galaxy and systematic velocity of the cluster). From this sample, we selected 2433 galaxies at $R<R_{200}$ as the cluster galaxy sample, and 6312 galaxies at $5 R_{200}<R<10 R_{200}$ as the field galaxy sample. Park \& Hwang (2009) showed that the fraction of earlytype galaxies decreases with increasing clustercentric radius at $R<3 R_{200}$. However, the early-type galaxy fraction is nearly constant at $R>4 R_{200}$ (see their Figure 4 ), suggesting that the galaxies at $5 R_{200}<R<10 R_{200}$ can be considered to be field galaxies. The galaxies in the two comparison samples also satisfy the criterion of $M_{r}<-19.77$ and $0.01<z<0.0741$ as do those in the V1 sample.

The optical parameters of galaxies that we consider in this study are $r$-band Petrosian absolute magnitude $\left(M_{r}\right), u-r$ color, and morphology. The $M_{r}$ was computed using the Galactic reddening correction (Schlegel et al. 1998), $K$-corrections (Blanton \& Roweis 2007), shifted to $z=0.1$, and evolution correction, $E(z)=1.6(z-0.1)$ (Tegmark et al. 2004). The $u-r$ color was computed using extinction- and $K$-corrected (to $z=0.1$ ) $u$ - and $r$-band model magnitudes. Galaxy morphology data are mainly from the Korea Institute for Advanced Study (KIAS) DR7 value-added galaxy cata$\log$ (Choi et al. 2010). Galaxies in this catalog are morphologically classified into early $(\mathrm{E} / \mathrm{S} 0)$ and late types ( $\mathrm{S} / \mathrm{Irr}$ ) based on an automated scheme with $u-r$ color, 


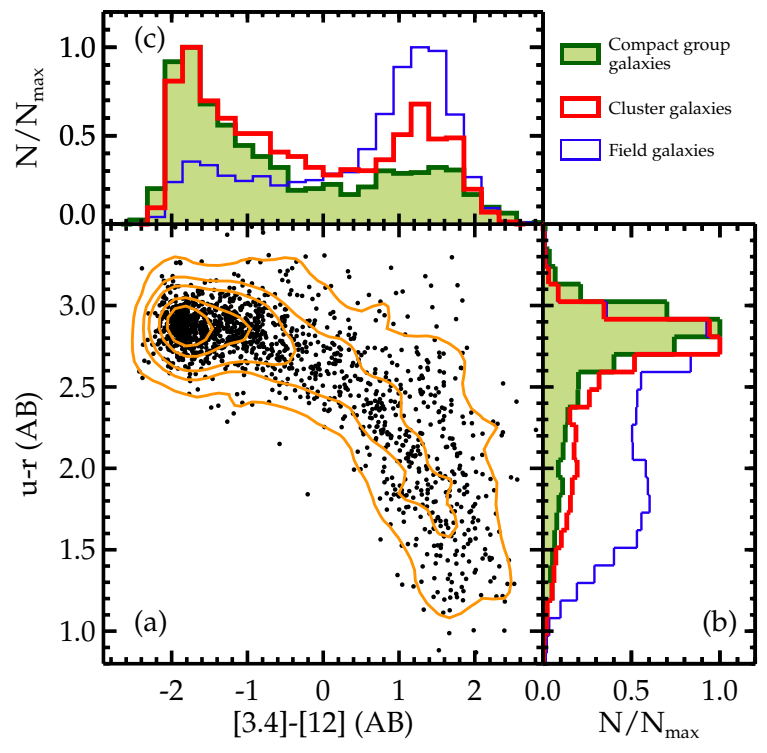

FIG. 1.- (a) $u-r$ versus [3.4] - [12] diagram for the 1541 compact group galaxies detected at $12 \mu \mathrm{m}$. Contours represent the number density distribution. In panels (b) and (c), we display the $u-r$ and [3.4] - [12] distribution for the compact group galaxies with the filled histograms. For comparison, we also plot the MIR color distribution of the 1461 cluster galaxies (open histogram with thick lines) and for the 5084 field galaxies (open histogram with thin lines).

the $g-i$ color gradient, and the $i$-band concentration index (Park \& Choi 2005). For galaxies not included in the KIAS DR7 value-added galaxy catalog, Sohn et al. (2016) classified them into early and late types through visual inspection. In Table 1, we list the group ID, galaxy ID, R.A., declination, redshift, $M_{r}, u-r$, and morphology of the 2175 compact group galaxies.

\section{RESULTS}

\subsection{Galaxy Color Distributions}

Figure 1 1 shows the $u-r$ and [3.4] - [12] distributions of the 1541 compact group galaxies detected at $12 \mu \mathrm{m}$. The mean $u-r$ error is 0.07 , while the mean [3.4] $-[12]$ error is 0.12 . In this diagram, compact group galaxies are distributed from the top-left corner to the bottom-right corner because galaxies with bluer optical colors tend to have redder MIR colors.

In panels (b) and (c), we plot the $u-r$ and [3.4] - [12] distributions of compact group galaxies. For comparison, we also plot the distributions of cluster and field galaxies. As shown in panel (b), $u-r$ histograms for the three galaxy samples peak at $u-r \simeq 2.6$. However, the fraction of galaxies with $u-r>2.5$ in the compact group galaxy sample is $73.8 \% \pm 1.1 \%$, which is larger than that of the cluster galaxy sample $(64.1 \% \pm 1.2 \%)$ and of the field galaxy sample $(38.4 \% \pm 0.7 \%)$. The mean $u-r$ is $2.65 \pm 0.02$ in the compact group galaxy sample, which is also larger than the $2.50 \pm 0.01$ of the cluster galaxy sample and the $2.22 \pm 0.01$ of the field galaxy sampl 3 . These suggest that compact group galaxies are dominated by optical red-sequence galaxies much like cluster galaxies, which is consistent with the findings of

3 The errors in the fractions and in the mean values are the standard deviations derived from 1000-times resamplings.

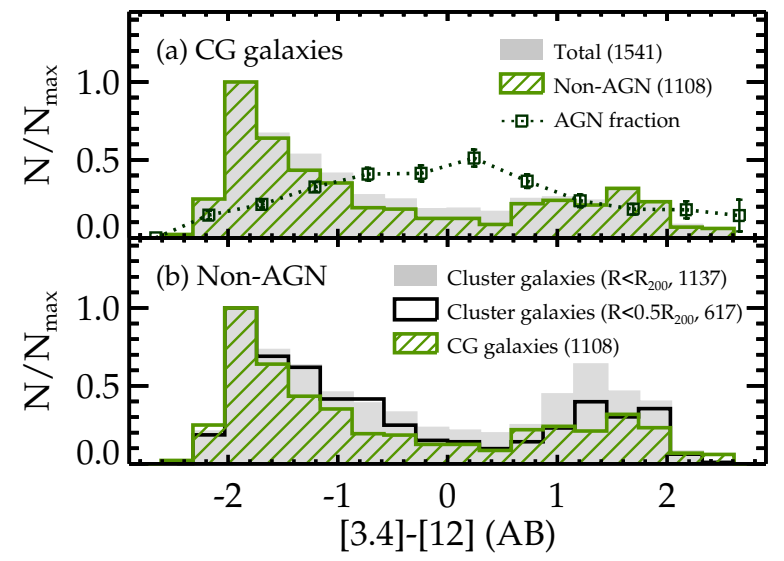

FIG. 2.- (a) [3.4] - [12] color distribution for the all 1541 (filled) and the 1108 non-AGN (hatched) compact group galaxies. Squares represent the fraction of AGN as a function of [3.4] - [12]. The error bars indicate the $1 \sigma$ deviation from 1000-times bootstrap resamplings. (b) Comparison of the color distributions between the 1108 non-AGN compact group galaxies (hatched), the 1137 non-AGN cluster galaxies at $R<R_{200}$ (filled), and the 617 nonAGN cluster galaxies at $R<0.5 R_{200}$ (open).

\section{Walker et al. (2013) and Zucker et al. (2016).}

Optical red-sequence galaxies, located in a narrow $u-r$ range of $2.5-3.2$, have MIR colors ranging from $[3.4]-[12] \simeq-2.4$ to $[3.4]-[12] \simeq 0$. Lee et al. (2015) divided optical red-sequence galaxies into MIR blue galaxies (i.e., [3.4] $-[12]<-1.55)$ and MIR green galaxies (i.e., $-1.55 \leq[3.4]-[12]<-0.3)$. The MIR blue galaxies are composed of stellar populations older than 10 Gyr, while the MIR green galaxies have small ( $\sim 5 \%)$ mass fractions of relatively young $(0.5-1$ Gyr) or intermediate $(1-10 \mathrm{Gyr})$ stellar populations (Piovan et al. 2003; Ko et al. 2013; Lee et al. 2015; Ko et al. 2016). Optical colors trace star formation on timescales up to 1 Gyr (Schawinski et al. 2014). Therefore, the MIR green galaxies are not distinguishable from the MIR blue galaxies in optical colors because both lie in the optical red-sequence (Walker et al. 2012, 2013; Ko et al. 2013; Lee et al. 2015; Zucker et al. 2016). Thus, we need to use MIR colors instead of optical colors to investigate whether galaxies in compact groups experienced different star formation histories from those in clusters, or not.

In panel (c) of Figure 1, we compare the MIR color distributions between the three different galaxy samples. The cluster galaxy sample shows a blue peak at $[3.4]-[12] \simeq-1.7$ and a red peak $[3.4]-[12] \simeq 1.3$. The blue peak is higher than the red peak. The compact group galaxy sample shows a strong blue peak and a red bump, but the field galaxy sample shows a different color distribution with a blue bump and a strong red peak. The mean [3.4] $-[12]$ is $-0.54 \pm 0.03$ for the compact group galaxy sample, $-0.29 \pm 0.04$ for the cluster galaxy sample, and $0.46 \pm 0.02$ for the field galaxy sample. These results suggest that compared to cluster galaxies, compact group galaxies are more dominated by

4 This criterion is determined from the decomposition of the [3.4] - [12] color distribution with three Gaussian functions using the Gaussian mixture modeling (Muratov \& Gnedin 2010, see Figure 2 in Lee et al. 2015). We stress that this MIR green color selection criterion is not identical to the "MIR green valley" one in Section 3.2. 
MIR blue galaxies: the fraction of MIR blue galaxies is $32.6 \% \pm 1.2 \%$ in the compact group galaxy sample and $23.7 \% \pm 1.1 \%$ in the cluster galaxy sample. This suggests that the mean stellar ages of compact group galaxies are on average older than those of cluster galaxies.

On the other hand, because compact groups favor strong galaxy interactions, the presence of active galactic nuclei (AGN) should be expected (Coziol et al. 1998a,b; Martínez et al. 2010; Tzanavaris et al. 2014). Because the contribution of AGN to the MIR fluxes of the host galaxies is not negligible, we identify the AGN in our sample and remove them. First, we identify optically selected AGN using the scheme given by Kewlev et al. (2006) who classified star-forming galaxies from AGN (Seyferts and LINERs) and composite galaxies in the $[\mathrm{NII}] / \mathrm{H} \alpha$ versus $[\mathrm{OII}] / \mathrm{H} \beta$ diagram (see their Figure 1 ). We remove $281 \mathrm{AGN}$ and 149 composite galaxies from the sample of 1541 compact group galaxies. Second, we identify MIR selected AGN using the classification method introduced by Mateos et al. (2012) who defined the AGN wedge in the WISE [3.4]-[4.6] versus [4.6] - [12] color-color diagram (see their Figure 2). We find 8 MIR selected AGN in the compact group galaxy sample. Among the 8 MIR selected AGN, 5 are also optically selected AGN. In total, 433 galaxies (28\%) are classified as AGN-host galaxies among the 1541 compact group galaxies.

We compare the MIR color distribution of all 1541 compact group galaxies with that of 1108 non-AGN compact group galaxies in Figure 2(a). The difference between the two distributions is remarkable at $-1.5 \lesssim$ $[3.4]-[12] \lesssim 0.5$ where the AGN fraction is larger than $30 \%$. This color range is similar to the WISE infrared transition zone, $-1.71 \lesssim[3.4]-[12] \lesssim 0.02$, defined by Alatalo et al. (2014)5. They showed that Seyferts and LINERs are representatives of the zone, which is consistent with our result.

In Figure 2(b), we compare the MIR color distribution of non-AGN compact group galaxies with that of non-AGN cluster galaxies. In this comparison, we use 1137 non-AGN cluster galaxies $\left(R<R_{200}\right)$ after removing 324 AGN-host galaxies (22\%) from the 1461 cluster galaxy sample. To statistically examine the difference in the color distributions of the two samples, we compute $p$-values from the Kolmogorov-Smirnov (KS) test $\left(P_{\mathrm{KS}}\right)$ and the Anderson-Darling (AD) k-sample test $\left(P_{\mathrm{AD}}\right)$. The $P_{\mathrm{KS}}$ and $P_{\mathrm{AD}}$ indicate the probability of rejecting the null hypothesis that the two samples are drawn from the same parent sample. The two tests on the two color distributions give the $P_{\mathrm{KS}}$ and $P_{\mathrm{AD}}$ of $\ll 0.01$, rejecting the null hypothesis at a significance of $>3 \sigma$ level.

In Figure 2(b), we use another comparison sample of 617 cluster galaxies located at $R<0.5 R_{200}$. In comparison with the $R<R_{200}$ cluster galaxy sample, the $R<0.5 R_{200}$ cluster galaxy sample displays the MIR color distribution more colsely to that of the compact group galaxy sample $\left(P_{\mathrm{KS}}=0.076\right.$ and $P_{\mathrm{AD}}=0.035$; the null hypothesis is rejected at the $<1.8 \sigma$ level). The fractions of MIR red (i.e., [3.4] - [12] $\geq-0.3$ ) galaxies in the compact group galaxy sample is $35.4 \% \pm 1.4 \%$. This is similar to the $34.7 \% \pm 1.8 \%$ in the $R<0.5 R_{200}$ cluster

5 This color range is converted from the $0.8<[4.6]-[12]<2.4$ in Vega magnitude in Alatalo et al. (2014). galaxy sample, but smaller than the $43.9 \% \pm 1.5 \%$ in the $R<R_{200}$ cluster galaxy sample. The mean [3.4] - [12] for the compact group galaxy sample $(-0.57 \pm 0.04)$ is similar to the $-0.52 \pm 0.06$ for the $R<0.5 R_{200}$ cluster galaxy sample, but smaller than the $-0.30 \pm 0.04$ for the $R<R_{200}$ cluster galaxy sample. The earlytype galaxy fraction in the $R<0.5 R_{200}$ cluster galaxy sample $(66.0 \% \pm 1.9 \%)$ is similar to that in the compact group galaxy sample $(62.0 \% \pm 1.5 \%)$, while the fraction is $57.5 \% \pm 1.5 \%$ in the $R<R_{200}$ cluster galaxy sample. These results suggest that compact groups are composed of galaxy populations similar to those in cluster central regions.

However, we find that the fraction of MIR green (i.e., $-1.55 \leq[3.4]-[12]<-0.3)$ galaxies is smaller in the compact group galaxy sample $(28.5 \% \pm 1.3 \%)$ than in the $R<0.5 R_{200}$ cluster galaxy sample $(34.5 \% \pm 1.9 \%)$. This may suggest that galaxy transition occurs faster in compact groups than in cluster environments. Hereafter, we use only the $R<0.5 R_{200}$ cluster galaxy sample in the comparison with the compact group galaxy sample.

Figure 3(a) shows again the MIR color distributions of the 1108 non-AGN compact group galaxies and of the 617 non-AGN cluster galaxies. In Figure 3 we divide these two galaxy samples into early-type and late-type galaxy samples. In Figure 3(b) we compare the MIR color distribution of the 687 compact group early-type galaxies with that of the 407 cluster early-type galaxies. The $P_{\mathrm{KS}}$ and $P_{\mathrm{AD}}$ values of $<0.001$ reject the null hypothesis for the two color distributions at a $>3 \sigma$ significance. We find that the fraction of MIR green galaxies is smaller in the compact group early-type galaxy sample $(37.6 \% \pm 1.8 \%)$ than in the cluster early-type galaxy sample $(47.4 \% \pm 2.5 \%)$, and that the mean [3.4] - [12] value is smaller in the compact group early-type galaxy sample $(-1.40 \pm 0.03)$ than in the cluster early-type galaxy sample $(-1.28 \pm 0.04)$.

In Figure 3(c) we compare the color distribution of the 421 compact group late-type galaxy sample with that of the 210 cluster late-type galaxy sample. The difference in the color distribution for the two late-type galaxy samples is not highly significant $(<1.9 \sigma)$, compared to the case for early-type galaxy samples. However, the mean $[3.4]-[12]$ value is smaller in the compact group late-type galaxy sample $(0.80 \pm 0.06)$ than in the cluster late-type galaxy sample $(0.95 \pm 0.07)$. The MIR green galaxy fraction is larger in the compact group late-type galaxy sample $(13.8 \% \pm 1.7 \%)$ than in the cluster late-type galaxy sample $(9.5 \% \pm 1.9 \%)$.

Because galaxy properties are dependent on their stellar masses (or luminosities), we compare the MIR color distributions of compact group galaxies and cluster galaxies by using a fixed $M_{r}$. We use $M_{r}$ instead of stellar masses because the former is available for all galaxies in both compact group and cluster galaxy samples. We find that the null hypothesis is rejected in the comparison for early-type galaxies with $-20.77 \geq M_{r}>-21.77$ at the $\gtrsim 2.2 \sigma$ level (panel h; $P_{\mathrm{KS}}=0.03$ and $P_{\mathrm{AD}}=0.001$ ). For the total galaxy sample with $-20.77 \geq M_{r}>-21.77$ (panel g), $P_{\mathrm{AD}}$ rejects the null hypothesis at the $2 \sigma$ level, but $P_{\mathrm{KS}}$ does not. The other cases show lower significance of rejection. This indicates that the difference in the MIR color distribution between the compact group galaxies and the cluster galaxies is mainly attributed to 


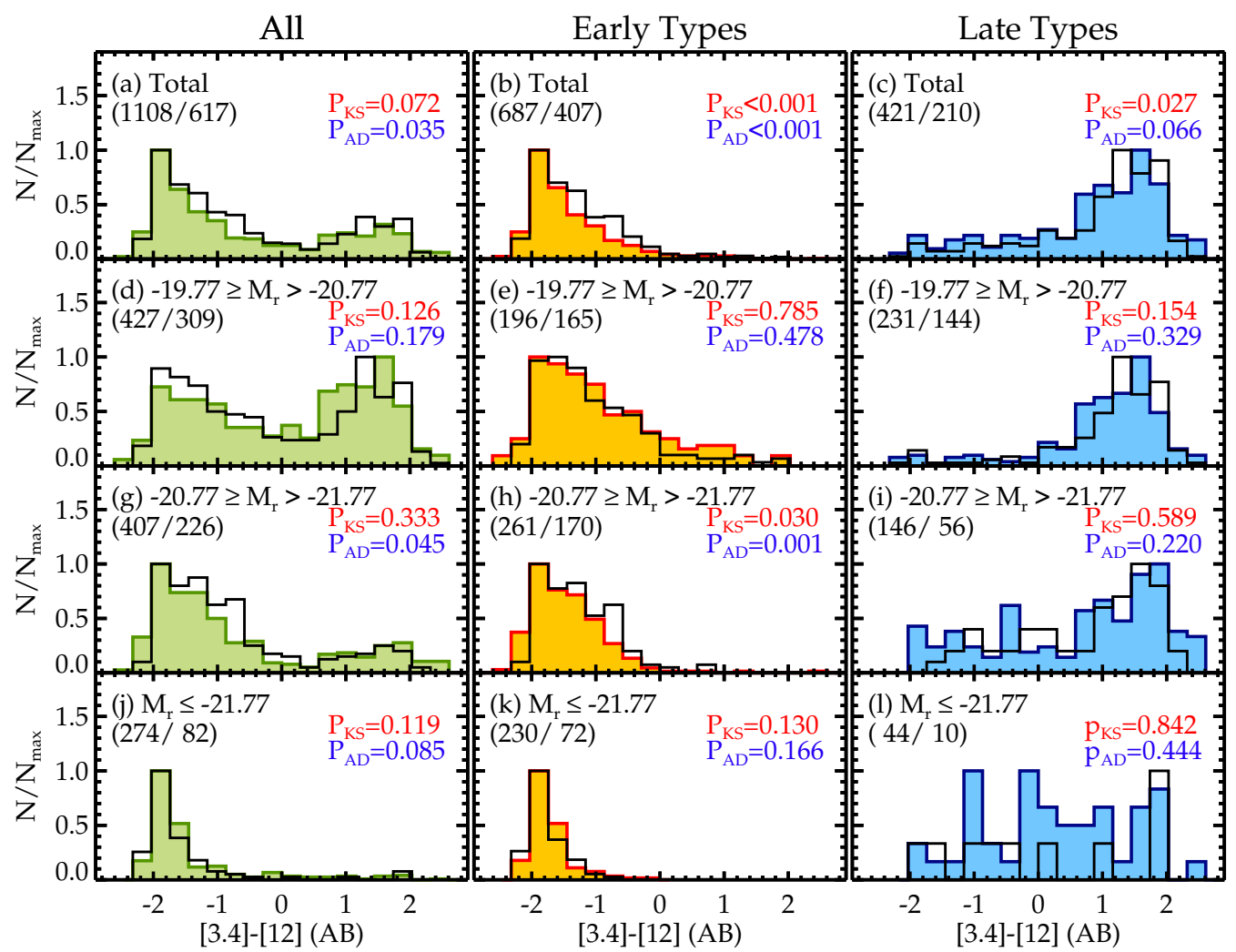

FIG. 3.- Comparison of [3.4] - [12] color distributions for compact group galaxies (filled histograms) with those of cluster galaxies (open histograms). The middle column is for early-type galaxies, and the right-hand column is for late-type galaxies. The left-hand column is the sum. The second row is for faint galaxies with $-19.77>M_{r}>-20.77$, the third row is for galaxies with $-20.77>M_{r}>-21.77$, and the bottom row is for bright galaxies with $M_{r} \leq-21.77$. We list $p$-values from the KS and AD k-sample tests for the two distributions (for compact group galaxies and for cluster galaxies) in each panel.

TABLE 2

WiSE-related Physical Parameters of Compact Group Galaxies

\begin{tabular}{ccrrrrcc}
\hline \hline Host Group & Galaxy ID & \multicolumn{1}{c}{$[3.4]-[12]^{a}$} & \multicolumn{1}{c}{$(\mathrm{S} / \mathrm{N})_{12}^{b}$} & \multicolumn{1}{c}{$\log \left(L_{12} / L_{\odot}\right)^{c}$} & $\mathrm{AGN}^{d}$ & $M_{3.4}^{e}$ & $\log \left(M_{\text {star }} / M_{\text {sun }}\right)^{f}$ \\
\hline V1CG001 & 1237648705657307198 & $-9.99 \pm 0.00$ & -9.9 & $-9.99 \pm 0.00$ & 0 & $-9.99 \pm 0.00$ & $0.00_{-0.00}^{+0.00}$ \\
V1CG001 & 1237648705657307347 & $0.00 \pm 0.00$ & 1.4 & $8.60 \pm 0.00$ & 0 & $-23.04 \pm 0.03$ & $9.92_{-0.05}^{+0.07}$ \\
V1CG001 & 1237648705657307315 & $-0.92 \pm 0.00$ & 0.2 & $8.50 \pm 0.00$ & 0 & $-23.71 \pm 0.04$ & $10.61_{-0.17}^{+0.12}$ \\
V1CG002 & 1237661126155436166 & $-0.87 \pm 0.05$ & 26.5 & $8.40 \pm 0.02$ & 1 & $-23.42 \pm 0.02$ & $10.70_{-0.15}^{+0.12}$ \\
V1CG002 & 1237661126155436169 & $0.69 \pm 0.03$ & 44.9 & $8.95 \pm 0.01$ & 1 & $-23.22 \pm 0.02$ & $10.63_{-0.10}^{+0.08}$ \\
V1CG002 & 1237661126155436164 & $-2.03 \pm 0.05$ & 23.2 & $8.61 \pm 0.02$ & 0 & $-25.10 \pm 0.02$ & $11.59_{-0.13}^{+0.07}$ \\
V1CG003 & 1237661136886431890 & $0.81 \pm 0.11$ & 9.3 & $8.71 \pm 0.05$ & 0 & $-22.51 \pm 0.03$ & $10.13_{-0.12}^{+0.09}$ \\
V1CG003 & 1237661136886497282 & $2.04 \pm 0.05$ & 31.7 & $9.67 \pm 0.01$ & 1 & $-23.67 \pm 0.04$ & $10.20_{-0.13}^{+0.09}$ \\
V1CG003 & 1237661136886497353 & $0.41 \pm 0.10$ & 10.6 & $8.74 \pm 0.04$ & 0 & $-23.00 \pm 0.03$ & $10.14_{-0.08}^{+0.08}$ \\
\hline
\end{tabular}

Noте. - The full table is available in the online journal. A portion is shown here for guidance regarding its form and content. ${ }^{a}$ The ALLWISE profile-fitting AB magnitudes. - 9.99 indicates no ALLWISE photometry.

${ }^{b}$ The signal-to-ratio at $12 \mu \mathrm{m}$. -9.9 indicates no $A L L W I S E$ photometry. When $(\mathrm{S} / \mathrm{N})_{12} \leq 3$, we assign zero errors to [3.4] - [12] and $\log \left(L_{12} / L_{\odot}\right)$.

${ }^{c} 12 \mu \mathrm{m}$ luminosities. -9.99 indicates no ALLWISE photometry.

$d$ AGN classification (0: non-AGN, 1: optical AGN, 2: MIR AGN, and 3: optical+MIR AGN)

e $3.4 \mu \mathrm{m}$ absolute magnitudes. $-9.99 \pm 0.000$ indicates no ALLWISE photometry.

$f$ Stellar masses calculated using $H_{0}=70 \mathrm{~km} \mathrm{~s}^{-1} \mathrm{Mpc}^{-1} .0 .00$ means no measurement. 


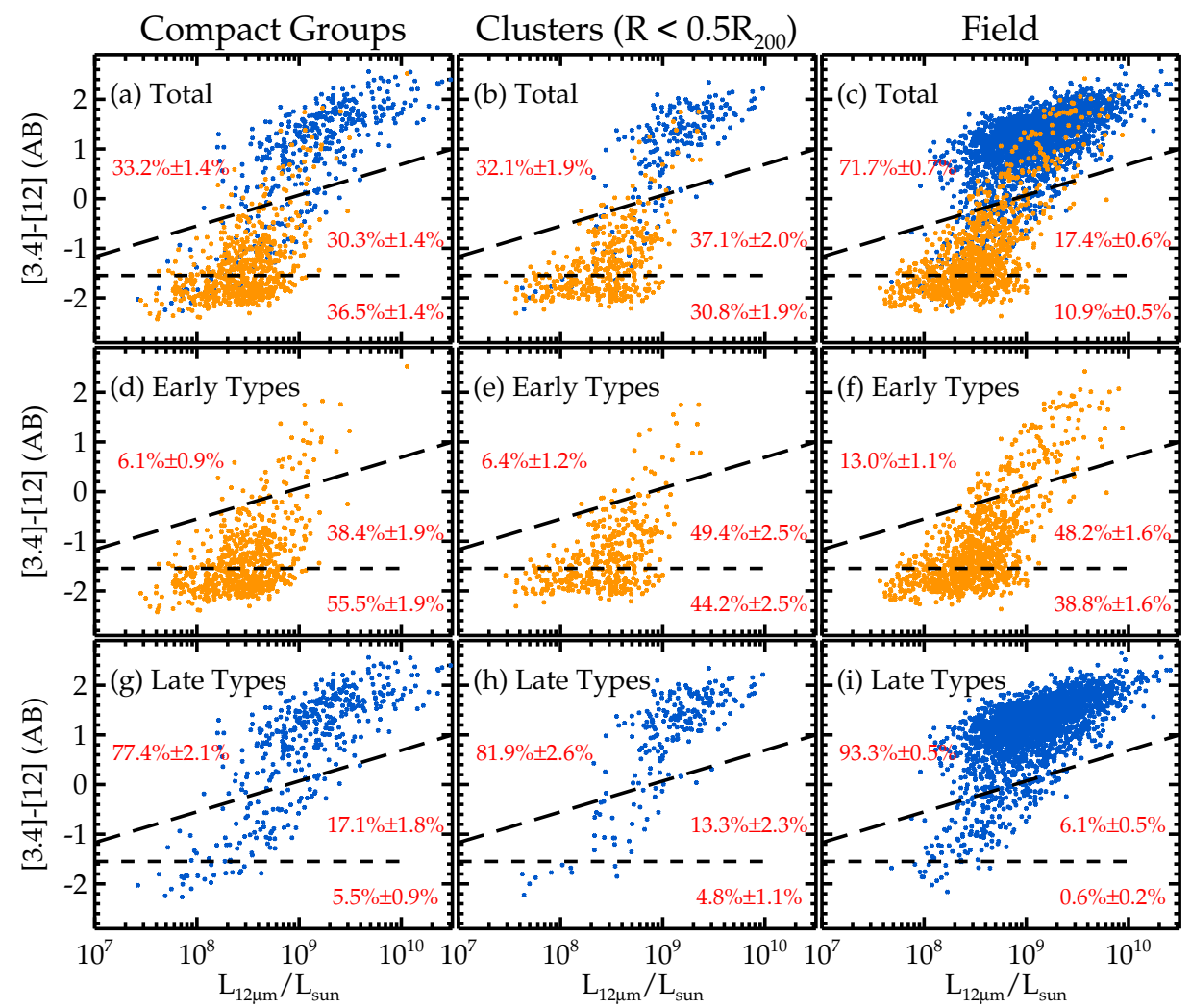

FIG. 4. - MIR color vs. luminosity diagrams for compact group galaxies (left), cluster $\left(R<0.5 R_{200}\right)$ galaxies (middle), and field galaxies (right). Orange and blue dots represent early- and late-type galaxies, respectively. We divide the galaxies into three classes following the classification scheme of Lee et al. (2015): MIR star-forming sequence galaxies (above the long-dashed lines), MIR blue cloud galaxies (below short-dashed lines), and MIR green valley galaxies (between the two lines). Middle and bottom rows are for early- and late-type galaxies in different environments. We list the fractions of different MIR classes in each panel. 
the galaxies with $-20.77 \geq M_{r}>-21.77$. We examine galaxy properties using the luminosity-limited subsamples in the following analysis, but find no significant difference from the results based on the total sample. We therefore present the results in the following analysis based on the total sample for better statistics.

\subsection{MIR Color-Luminosity Diagram}

In Figure 4we investigate the [3.4] - [12] versus $12 \mu \mathrm{m}$ luminosity distribution of galaxies in compact groups, clusters, and field. In the [3.4] - [12] versus $12 \mu \mathrm{m} \mathrm{lu-}$ minosity diagram, galaxies are divided into three classes following the classification scheme of Lee et al. (2015): MIR star-forming sequence galaxies (above the inclined dashed line:6]), MIR blue cloud galaxies ([3.4] - [12] < $-1.55)$, and MIR green valley galaxies located between the two classes. In this section we use this classification scheme to calculate the fractions of different MIR classes. In particular, MIR green valley galaxies are not necessarily "MIR green" in the sense of Section 3.1.

In the three panels in the top row of Figure 4, we plot 1108 compact group galaxies, 617 cluster galaxies, and 3854 field galaxies, separately. All these galaxies are not AGN-host galaxies. We find that the fraction of MIR blue cloud galaxies is larger in the compact group galaxy sample $(36.5 \% \pm 1.4 \%)$ than in the cluster galaxy sample $(30.8 \% \pm 1.9 \%)$ and in the field galaxy sample $(10.9 \% \pm 0.5 \%)$. In the cluster galaxy sample and the field galaxy sample, the fractions of MIR blue cloud galaxies are smaller than the fractions of MIR green valley galaxies. In contrast, in the compact group galaxy sample, the fraction of MIR blue cloud galaxies is larger than the fraction of MIR green valley galaxies $(30.3 \% \pm 1.4 \%)$.

When we consider only early-type galaxies, as shown in the middle row of Figure 4 , we obtain similar results. The fraction of early-type galaxies is $62.0 \%(687 / 1108)$ in the compact group galaxy sample, $66.0 \%(407 / 617)$ in the cluster galaxy sample, and $26.5 \%(1023 / 3854)$ in the field galaxy sample. Among the three early-type galaxy samples, the compact group early-type galaxy sample shows the highest MIR blue cloud galaxy fraction. In addition, in the cluster early-type galaxy sample and in the field early-type galaxy sample, the fractions of MIR blue cloud galaxies is smaller than those of MIR green valley galaxies. However, in the compact group early-type galaxy sample, the fraction of MIR blue cloud galaxies $(55.5 \% \pm 1.9 \%)$ is significantly larger than the fraction of MIR green valley galaxies $(38.4 \% \pm 1.9 \%)$. These results suggest not only that early-type galaxies in compact groups are on average older than those in other environments, but also that the timescales for early-type galaxies to stay in the MIR green valley are shorter in compact groups than in other environments.

In the bottom row of Figure 4 , we plot only late-type galaxies. The fraction of MIR star-forming sequence galaxies decreases from the field $(93.3 \% \pm 0.5 \%)$ to clusters $(81.9 \% \pm 2.6 \%)$ to compact groups $(77.4 \% \pm 2.1 \%)$. Thus, the fractions of MIR blue cloud galaxies and of MIR green valley galaxies are smallest in the field $(0.6 \% \pm 0.2 \%$ and $6.1 \% \pm 0.5 \%)$ and largest in compact groups $(5.5 \% \pm 0.9 \%$ and $17.1 \% \pm 1.8 \%)$. The smallest

$6 \quad[3.4]-[12]=\log \left(L_{12} / L_{\text {sun }}\right) \times 0.62-5.51$. See Section 5.1 of Lee et al. (2015) for details. fraction of MIR star-forming sequence galaxies in compact groups is consistent with the case that the star formation quenching for late-type galaxies occurs more effectively in compact groups than in clusters and in the field. We note that the results in this section do not change significantly if we use $-1.55 \leq[3.4]-[12]<-0.3$ as the criterion to select MIR green galaxies. In Table 2 , we list the WISE-related parameters and AGN classes of compact group galaxies.

\subsection{Environments of Compact Group Galaxies}

To investigate the environmental effects on galaxy properties in compact groups, we use three kinds of environmental parameters. The first parameter is the number of neighboring galaxies $\left(N_{\text {nei }}\right)$ of compact groups. Sohn et al. (2016) calculated $N_{\text {nei }}$ around compact groups within a comoving cylinder defined by projected group centric radius $R=1 \mathrm{Mpc}$ and $\Delta V=$ $\left|V_{\text {gal }}-V_{\text {gr }}\right|=1000 \mathrm{~km} \mathrm{~s}^{-1}$, where $V_{\text {gal }}$ and $V_{\text {gr }}$ are a radial velocity of a galaxy and a mean velocity of member galaxies in a compact group. In the $N_{\text {nei }}$ count, Sohn et al. (2016) used galaxies in the V2 sample $\left(M_{r}<-20.77\right.$ and $0.01<z<0.115)$. In this study, we recalculate $N_{\text {nei }}$ for 670 compact groups using galaxies in the V1 sample $\left(M_{r}<-19.77\right.$ and $\left.0.01<z<0.0741\right)$. We exclude the compact group members in the $N_{\text {nei }}$ count. We do not assign $N_{\text {nei }}$ to 59 compact groups close to the lower and upper redshift limits because their $N_{\text {nei }}$ can be underestimated, resulting in that we assign $N_{\text {nei }}$ to 611 compact groups.

The second environmental parameter is the fraction of early-type member galaxies in compact groups $\left(f_{\mathrm{E}}\right)$. Bitsakis et al. (2010, 2011, 2015) used this $f_{\mathrm{E}}$ to divide compact groups into dynamically old and young systems based on the assumption that dynamically old systems are dominated by early-type galaxies that could form through repeated interactions between member galaxies. Thus, $f_{\mathrm{E}}$ is the parameter reflecting the internal environment of compact groups.

The third environmental parameter is the rest-frame velocity dispersions of compact group member galaxies $\left(\sigma_{\mathrm{CG}}\right)$. We adopt the $\sigma_{\mathrm{CG}}$ from Table 5 in Sohn et al. (2016). Sohn et al. (2016) found that compact groups with low $\sigma_{\mathrm{CG}}\left(\lesssim 100 \mathrm{~km} \mathrm{~s}^{-1}\right)$ show features of ongoing interactions among member galaxies, but that compact groups with $\sigma_{\mathrm{CG}} \gtrsim 300 \mathrm{~km} \mathrm{~s}^{-1}$ do not. Thus, $\sigma_{\mathrm{CG}}$ is another parameter that reflects the internal environment of compact groups.

We note that we use all the member galaxies regardless of $12 \mu \mathrm{m}$ detection when we calculate $f_{\mathrm{E}}$ and $\sigma_{\mathrm{CG}}$. In Table 3, we list the ID, R.A., declination, redshift, as well as three environmental parameters of the 670 compact groups. Figure 5 shows the relations between the three environmental parameters. We plot only 611 systems with complete $N_{\text {nei }}$. The $N_{\text {nei }}$ histogram peaks at $N_{\text {nei }} \simeq$ $3-6$, and stretches out to more than $N_{\text {nei }}=40$. The $f_{\mathrm{E}}$ mainly has discontinuous values (e.g., $0,1 / 3,2 / 3$, and 1 ) because the majority $(495 / 611,81 \%)$ of our systems have three member galaxies. The $\sigma_{\mathrm{CG}}$ histogram peaks at $\sim 80 \mathrm{~km} \mathrm{~s}^{-1}$, and stretches out to more than $600 \mathrm{~km}$ $\mathrm{s}^{-1}$. The median $\sigma_{\mathrm{CG}}$ is $\sim 200 \mathrm{~km} \mathrm{~s}^{-1}$.

\subsubsection{Environmental Dependence on $N_{\text {nei }}$}


TABLE 3

Environmental Parameters of Compact Groups

\begin{tabular}{cccccccc}
\hline \hline Group ID $^{a}$ & R.A. $^{a}$ & Decl. $^{a}$ & Redshift $^{a}$ & $N_{\text {mem }}^{b}$ & $N_{\text {nei }}^{c}$ & $f_{\mathrm{E}}^{d}$ & $\sigma_{\mathrm{CG}}\left(\mathrm{km} \mathrm{s}^{-1}\right)^{e}$ \\
\hline V1CG001 & 198.227173 & 1.012775 & $0.0723 \pm 0.0008$ & 3 & -99 & 0.67 & $482 \pm 122$ \\
V1CG002 & 139.935760 & 33.744308 & $0.0202 \pm 0.0012$ & 3 & 11 & 0.67 & $866 \pm 228$ \\
V1CG003 & 154.741531 & 37.298065 & $0.0480 \pm 0.0003$ & 3 & 5 & 0.00 & $210 \pm 37$ \\
V1CG004 & 158.222275 & 12.086633 & $0.0330 \pm 0.0004$ & 3 & 11 & 0.67 & $242 \pm 69$ \\
V1CG005 & 127.709404 & 28.573534 & $0.0657 \pm 0.0001$ & 3 & 1 & 0.33 & $26 \pm 4$ \\
V1CG006 & 149.408493 & 37.324886 & $0.0611 \pm 0.0002$ & 3 & 4 & 0.33 & $100 \pm 26$ \\
V1CG007 & 240.816635 & 45.356598 & $0.0420 \pm 0.0002$ & 3 & 3 & 0.33 & $89 \pm 15$ \\
V1CG008 & 239.193481 & 46.345490 & $0.0420 \pm 0.0009$ & 3 & 3 & 1.00 & $483 \pm 83$ \\
V1CG009 & 204.321213 & 45.249256 & $0.0620 \pm 0.0003$ & 3 & 7 & 0.67 & $189 \pm 47$ \\
V1CG010 & 161.923065 & 38.933136 & $0.0360 \pm 0.0004$ & 4 & 14 & 0.50 & $272 \pm 75$ \\
\hline
\end{tabular}

NotE. - The full table is available in the online journal. A portion is shown here for guidance regarding its form and content.

$a$ Group ID from Table 5 of Sohn et al. (2016).

${ }^{b}$ The number of member galaxies in each compact group.

${ }^{c}$ The number of neighboring galaxies $\left(M_{r}<-19.77\right)$ in the comoving cylinder. For 59 compact groups near the lower and upper redshift limits, $N_{\text {nei }}$ are not assigned $\left(N_{\text {nei }}=-99\right)$.

$d$ The fraction of early-type galaxies among member galaxies of a compact group.

$e$ The rest-frame line-of-sight velocity dispersion of galaxies in each compact group and the $1 \sigma$ error derived from 1000-times bootstrap resamplings, from Table 5 of Sohn et al. (2016).

To examine the effects of $N_{\text {nei }}$ on galaxy properties in compact groups, we investigate how the MIR color distribution of compact group galaxies varies with $N_{\text {nei }}$. Figure 6 shows the MIR color histograms of the galaxies in different $N_{\text {nei }}$ bins. The left-hand column shows that the fraction of MIR red (i.e., [3.4] - [12] > -0.3) galaxies dramatically decreases as $N_{\text {nei }}$ increases. The MIR red galaxies are mainly late-type galaxies. The fraction of late-type galaxies gradually decreases from $56.2 \% \pm 3.0 \%$ (panel a) to $15.9 \% \pm 2.6 \%$ (panel d) as $N_{\text {nei }}$ increases. For comparison, we also plot the MIR color distribution of cluster galaxies as open histograms. We find that the fraction of late-type galaxies in the $N_{\text {nei }} \geq 8$ compact groups is smaller than the fraction of late-type galaxies $(34.0 \% \pm 1.9 \%)$ in the cluster galaxy sample.

We investigate the MIR color distributions for earlyand late-type galaxies separately in the middle and right

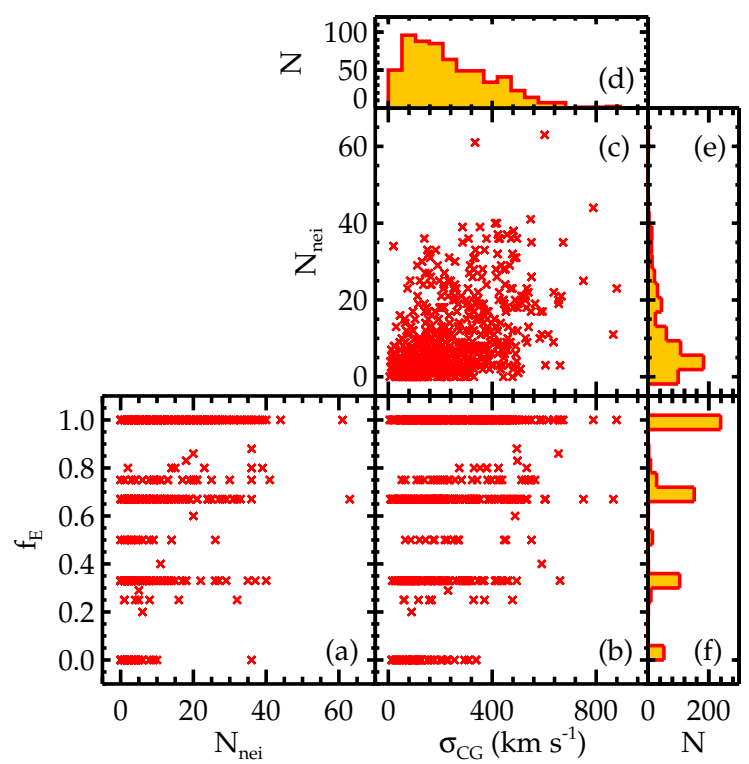

FIG. 5.- Relation between the three environmental parameters for compact groups: (a) $f_{\mathrm{E}}$ vs. $N_{\text {nei }}$, (b) $f_{\mathrm{E}}$ vs. $\sigma_{\mathrm{CG}}$, and (c) $N_{\text {nei }}$ vs. $\sigma_{\mathrm{CG}}$ diagrams. $(\mathrm{d}-\mathrm{f})$ Histograms for the three parameters. columns in Figure 6 The KS and AD k-sample tests for early-type galaxy samples in the four different $N_{\text {nei }}$ bins $(\mathrm{e}-\mathrm{h})$ reject the null hypothesis at the $<1.3 \sigma$ level. They also reject the null hypothesis for late-type galaxy samples in the four different $N_{\text {nei }}$ bins (i-l) at the $<1.8 \sigma$ level. These imply that $N_{\text {nei }}$ does not affect the MIR colors of early- and late-type galaxies in compact groups directly. Thus, the $N_{\text {nei-dependence of MIR colors shown }}$ in the left column $(\mathrm{a}-\mathrm{d})$ results from the fact that compact groups located in denser regions (larger $N_{\text {nei }}$ ) tend to have more early-type member galaxies with MIR blue colors.

The middle column in Figure 6 shows that compact group early-type galaxies have MIR colors bluer than cluster early-type galaxies regardless of $N_{\text {nei }}$. The color difference is significant $(\gtrsim 2.1 \sigma)$ in the $N_{\text {nei }}<20$ compact groups, but it is marginal $(\sim 1.5 \sigma)$ in the $N_{\text {nei }} \geq 20$ groups. We find no color difference between cluster latetype galaxies and compact group late-type galaxies at the $N_{\text {nei }}<8$ groups. However, late-type galaxies at the $N_{\text {nei }} \geq 8$ groups have MIR colors bluer than cluster latetype galaxies. The significance of the color difference is $\sim 2-3 \sigma$.

\subsubsection{Environmental Dependence on $f_{\mathrm{E}}$}

Figure 7 shows the MIR color distributions of compact group early-type galaxies in different $f_{\mathrm{E}}$ bins: $f_{\mathrm{E}}<0.5$, $0.5 \leq f_{\mathrm{E}}<1$, and $f_{\mathrm{E}}=1$. We find no significant differences in the MIR colors between the three $f_{\mathrm{E}}$ bins. The mean [3.4] - [12] value is $-1.49 \pm 0.08$ at $f_{\mathrm{E}}<0.5$, $-1.44 \pm 0.04$ at $0.5 \leq f_{\mathrm{E}}<0.1$, and $-1.43 \pm 0.03$ at $f_{\mathrm{E}}=1$, respectively. These mean values agree well within $1 \sigma$ errors. The KS and the AD k-sample tests do not reject the null hypothesis, either.

Bitsakis et al. (2011) showed that early-type galaxies in dynamically young $\left(f_{\mathrm{E}}<0.25\right)$ compact groups have bluer NUV $-r$ colors than those in dynamically old $\left(f_{\mathrm{E}}>0.25\right)$ groups. However, the number of early-type galaxies in their dynamically young groups is only three. In our sample, there are no early-type galaxies belonging to the $f_{\mathrm{E}}<0.25$ groups, and only six early-type galaxies belonging to the $f_{\mathrm{E}}<0.3$ groups. However, we find 


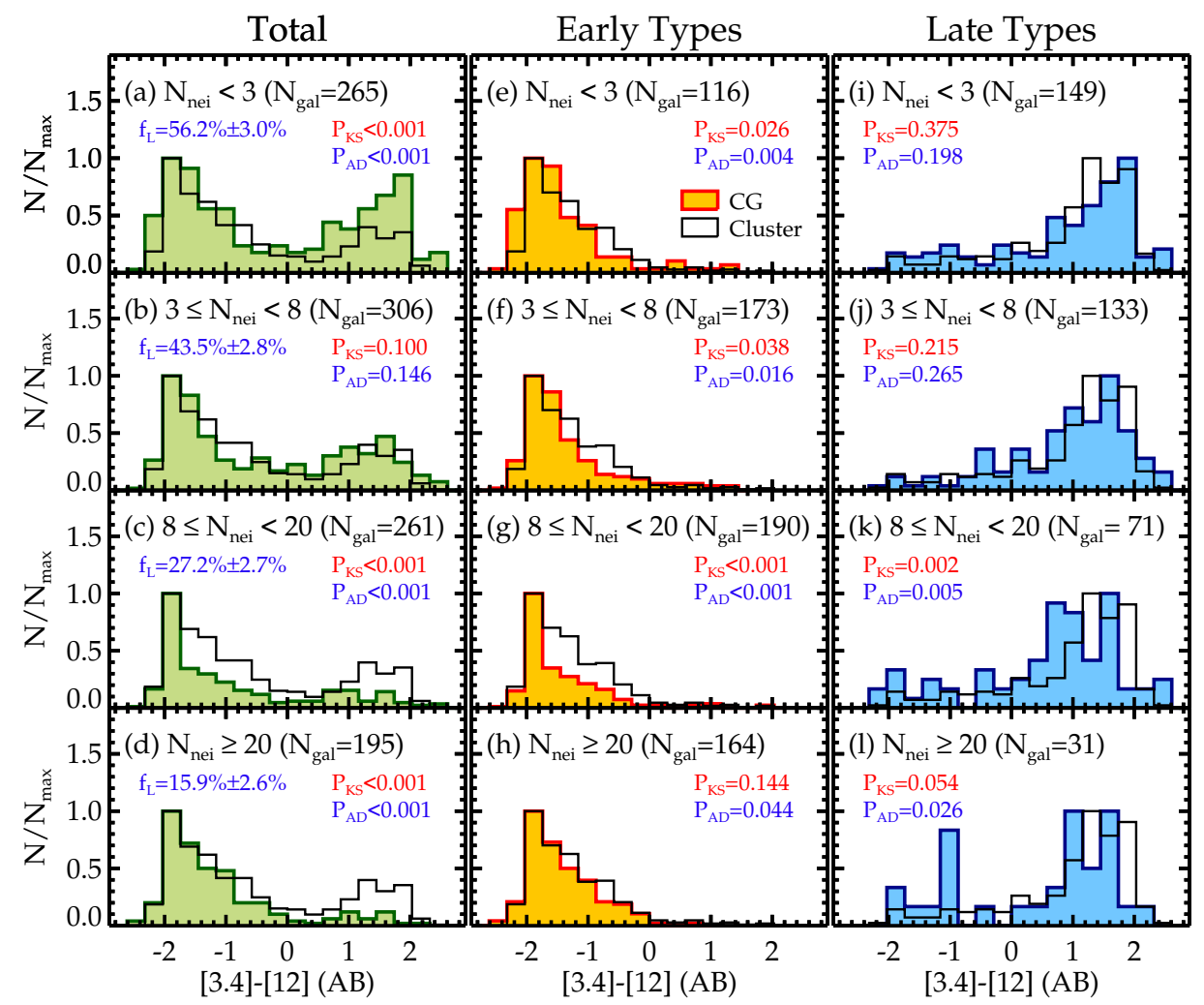

FIG. 6.- Dependence of the [3.4] - [12] color of compact group galaxies on the number of neighboring galaxies $\left(N_{\text {nei }}\right)$. In the left-hand column, we list the fraction of late-type galaxies $\left(f_{\mathrm{L}}\right)$. In the middle and right-hand columns, we plot early-type galaxies and late-type galaxies, separately. For comparison, we also plot the color distributions for cluster galaxies (open histograms).

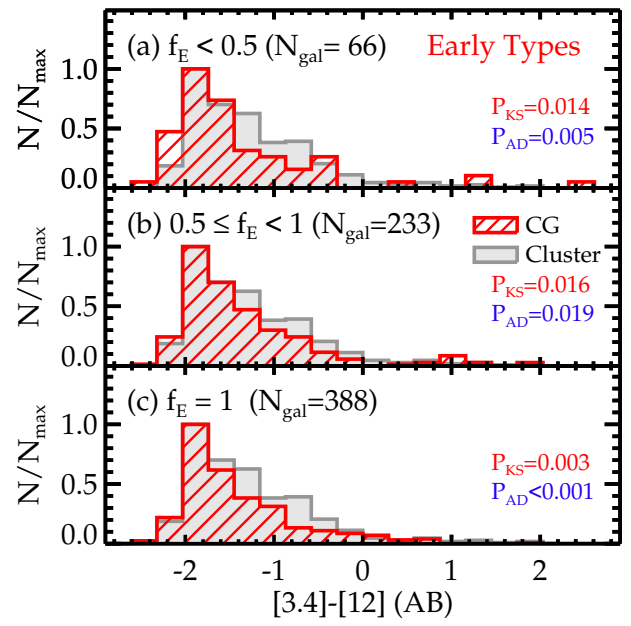

FIG. 7.- MIR color distributions for early-type galaxies in the (a) $f_{\mathrm{E}}<0.5$, (b) $0.5 \leq f_{\mathrm{E}}<1$, and (c) $f_{\mathrm{E}}=1$ compact groups. For comparison, we also plot the (filled) histograms for the early-type galaxies in our cluster sample.

that the MIR color distribution for the six galaxies is not different significantly from that for early-type galaxies in the $f_{\mathrm{E}}<0.5$ groups.

On the other hand, we find that in all three $f_{\mathrm{E}}$ bins, compact group early-type galaxies have MIR colors bluer than cluster early-type galaxies. The $P_{\mathrm{KS}}$ and $P_{\mathrm{AD}}$ are $<0.019$, suggesting that the color difference between compact group and cluster early-type galaxy samples is significant $(>2.4 \sigma)$. This is consistent with the result in Figure 6.

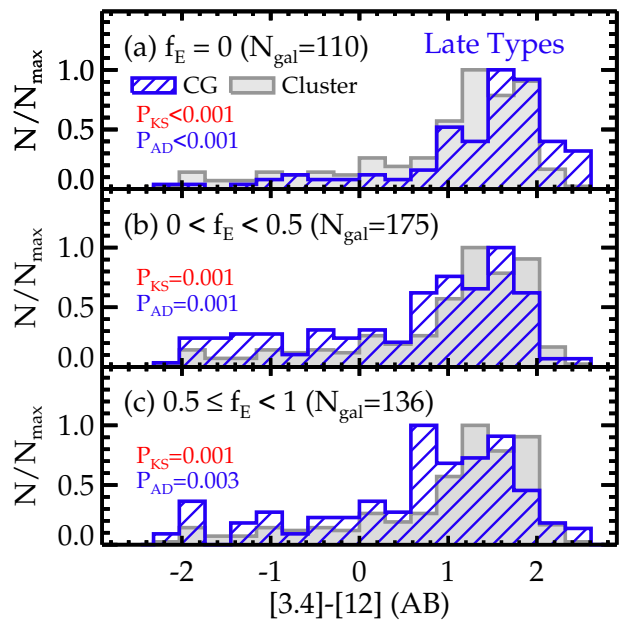

FIG. 8.- MIR color distributions for late-type galaxies in the (a) $f_{\mathrm{E}}=0$, (b) $0<f_{\mathrm{E}}<0.5$, and (c) $0.5<f_{\mathrm{E}}<1$ compact groups. For comparison, we also plot the (filled) histograms for the late-type galaxies in our cluster sample.

Figure 8 shows the MIR color distributions of compact group late-type galaxies in different $f_{\mathrm{E}}$ bins: $f_{\mathrm{E}}=0,0<$ $f_{\mathrm{E}}<0.5$, and $0.5 \leq f_{\mathrm{E}}<1$. For comparison, we also plot the color distributions for the cluster late-type galaxy sample. Late-type galaxies in the $f_{\mathrm{E}}=0$ compact groups have MIR colors redder than cluster late-type galaxies. However, late-type galaxies in the $f_{\mathrm{E}}>0$ groups have MIR colors bluer than cluster late-type galaxies. In all three bins, $P_{\mathrm{KS}}$ and $P_{\mathrm{AD}}$ are $\leq 0.03$, indicating that the color differences are significant $(>3 \sigma)$. On the other 


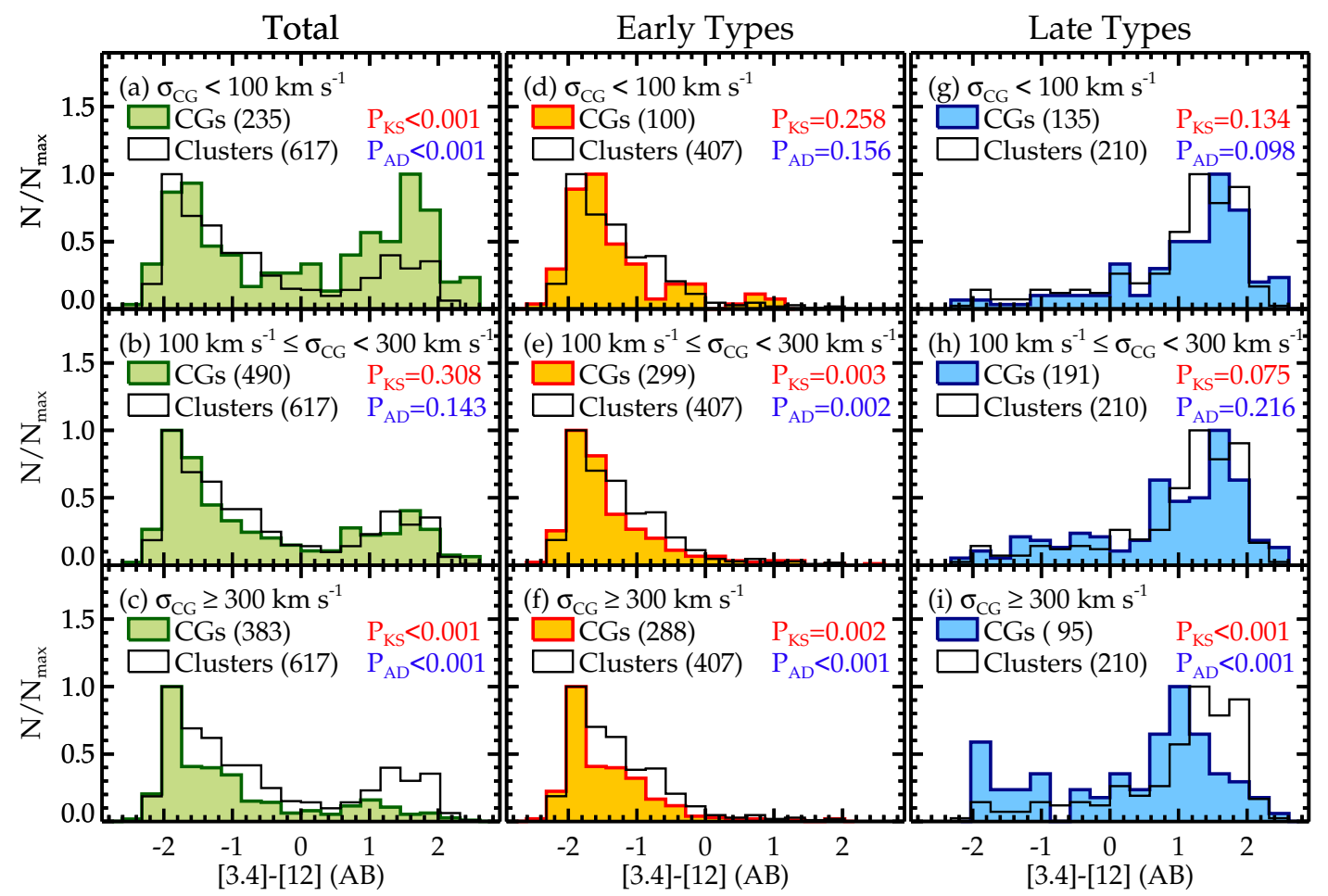

FIG. 9. - Left: MIR color distributions for compact group galaxies (filled histograms) in the (a) $\sigma_{\mathrm{CG}}<100 \mathrm{~km} \mathrm{~s}{ }^{-1}$, (b) $100 \mathrm{~km}$ $\mathrm{s}^{-1} \leq \sigma_{\mathrm{CG}}<300 \mathrm{~km} \mathrm{~s}^{-1}$, and (c) $\sigma_{\mathrm{CG}} \geq 300 \mathrm{~km} \mathrm{~s}^{-1}$ compact groups. Open histograms represent cluster galaxies. We plot early-type galaxies in the middle column $(\mathrm{d}-\mathrm{f})$ and late-type galaxies in the right column $(\mathrm{g}-\mathrm{i})$.

hand, we find that MIR colors of late-type galaxies in the $0<f_{\mathrm{E}}<0.5$ groups are not different from those in the $0.5 \leq f_{\mathrm{E}}<1$ groups $\left(P_{\mathrm{KS}}=0.835\right.$ and $\left.P_{\mathrm{AD}}=0.664\right)$. The mean [3.4] - [12] values of late-type galaxies in the $0<f_{\mathrm{E}}<0.5$ groups $(0.58 \pm 0.09)$ and in the $0.5 \leq f_{\mathrm{E}}<1$ groups $(0.64 \pm 0.10)$ are similar, but smaller than the $1.32 \pm 0.09$ in the $f_{\mathrm{E}}=0$ groups.

These results suggest that star formation activity of late-type galaxies is suppressed more efficiently in the $f_{\mathrm{E}}>0$ compact groups than in clusters. However, latetype galaxies in the $f_{\mathrm{E}}=0$ groups have comparable or higher star formation activity than that of cluster latetype galaxies. It is interesting that late-type member galaxies in compact groups show a hint of star formation quenching only when the compact groups have early-type member galaxies (see Section 4.3 for detailed discussion).

\subsubsection{Environmental Dependence on $\sigma_{\mathrm{CG}}$}

Figure 9 shows how the MIR colors of compact group galaxies depend on $\sigma_{\mathrm{CG}}$. We divide compact groups into low $\left(<100 \mathrm{~km} \mathrm{~s}^{-1}\right)$, intermediate $(100-300 \mathrm{~km}$ $\left.\mathrm{s}^{-1}\right)$, and high $\left(\geq 300 \mathrm{~km} \mathrm{~s}^{-1}\right) \sigma_{\mathrm{CG}}$ systems. In the left column, we find that the fraction of MIR red (i.e., $[3.4]-[12]>-0.3)$ galaxies decreases as $\sigma_{\mathrm{CG}}$ increases. This is because that the fraction of late-type galaxies is smaller in higher $\sigma_{\mathrm{CG}}$ systems. This trend is similar to the $N_{\text {nei-dependence in Figure } 6 \text {. }}$

The MIR colors of compact group early-type galaxies do not depend on $\sigma_{\mathrm{CG}}$. The MIR color distribution of early-type galaxies in the $\sigma_{\mathrm{CG}}<100 \mathrm{~km} \mathrm{~s}^{-1}$ groups does not differ from that of cluster early-type galaxies. However, the MIR colors of early-type galaxies in the $\sigma_{\mathrm{CG}} \geq 100 \mathrm{~km} \mathrm{~s}^{-1}$ groups are significantly $(>3 \sigma)$ differ- ent (bluer) from those of cluster early-type galaxies.

The MIR colors of late-type galaxies are bluer in the $\sigma_{\mathrm{CG}} \geq 300 \mathrm{~km} \mathrm{~s}^{-1}$ groups than in the $\sigma_{\mathrm{CG}}<300 \mathrm{~km} \mathrm{~s}^{-1}$ groups. The $P_{\mathrm{KS}}$ and $P_{\mathrm{AD}}$ confirm the color difference with a significance $\gtrsim 3 \sigma$ level. Furthermore, the MIR colors of late-type galaxies in the $\sigma_{\mathrm{CG}} \geq 300 \mathrm{~km} \mathrm{~s}^{-1}$ groups are significantly $(>4 \sigma)$ bluer than those of cluster late-type galaxies.

\subsubsection{Which Environment Most Affects the MIR Properties of Compact Group Galaxies?}

In Figures 6-9, we find that the MIR colors of compact group early-type galaxies do not depend much on the three environmental parameters, and that compact group late-type galaxies depend on $f_{\mathrm{E}}$ and $\sigma_{\mathrm{CG}}$, but not on $N_{\text {nei }}$. In $f_{\mathrm{E}}>0$ or $\sigma_{\mathrm{CG}} \geq 300 \mathrm{~km} \mathrm{~s}^{-1}$ compact groups, late-type member galaxies show bluer MIR color distributions. Bitsakis et al. (2011) showed that $\sigma_{\mathrm{CG}}$ tend to be larger in dynamically old $\left(f_{\mathrm{E}}>0.25\right)$ compact groups than in dynamically young groups. However, $f_{\mathrm{E}}$ and $\sigma_{\mathrm{CG}}$ for our compact groups do not have a significant correlation (Figure 5). Spearman's rank correlation coefficient for $f_{\mathrm{E}}$ and $\sigma_{\mathrm{CG}}$ is 0.28 and the probability of obtaining the correlation by chance is $\ll 0.001 \%$, suggesting that the correlation between the two parameters is weak.

To examine which environmental parameter among $f_{\mathrm{E}}$, $\sigma_{\mathrm{CG}}$, and $N_{\text {nei }}$ most affects the MIR colors of galaxies, we first consider $f_{\mathrm{E}}$ and $\sigma_{\mathrm{CG}}$ simultaneously in Figure 10. In the left panels we compare MIR colors of latetype galaxies in the $f_{\mathrm{E}}=0$ compact groups with those of late-type galaxies in the $f_{\mathrm{E}}>0$ groups in three $\sigma_{\mathrm{CG}}$ bins. We find that MIR colors of late-type galaxies in the $f_{\mathrm{E}}>0$ groups are bluer than those of late-type galaxies 


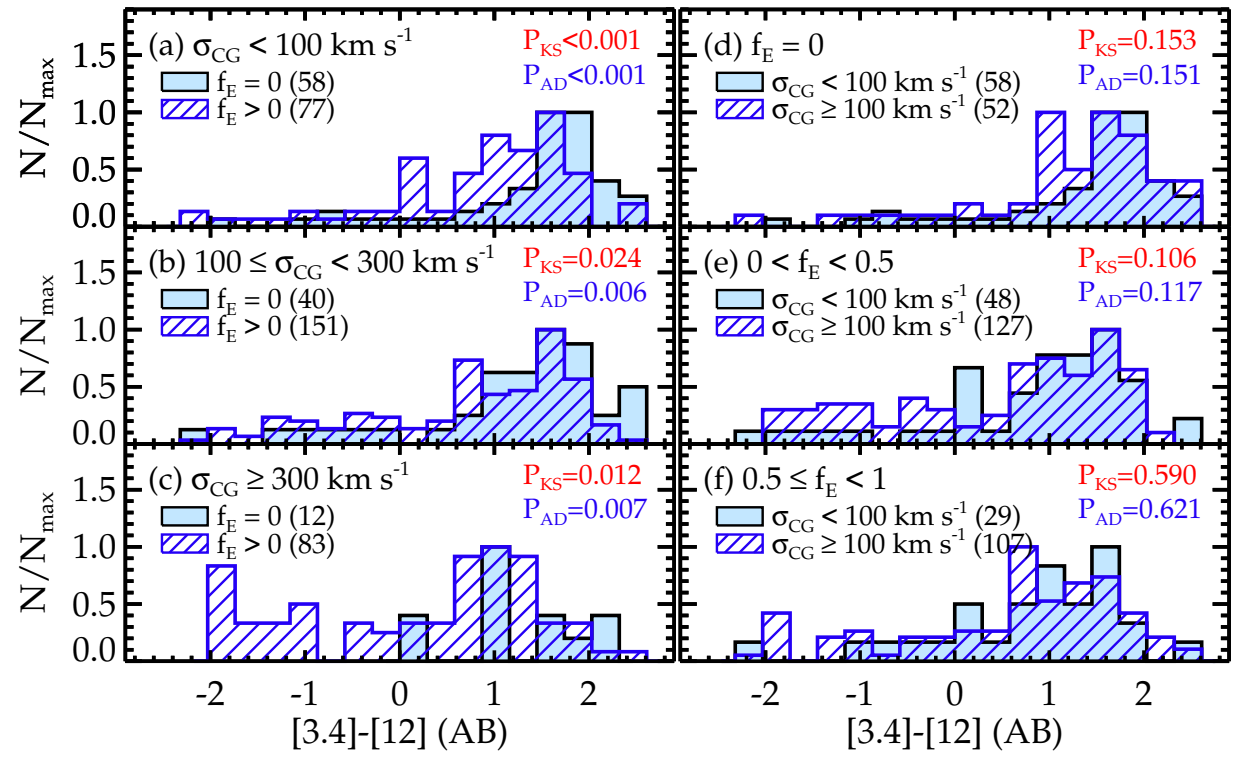

Fig. 10. - Left: MIR color distributions for late-type galaxies in the (a) $\sigma_{\mathrm{CG}}<100 \mathrm{~km} \mathrm{~s}^{-1}$, (b) $100 \leq \sigma_{\mathrm{CG}}<300 \mathrm{~km} \mathrm{~s}-1$, and (c) $\sigma_{\mathrm{CG}} \geq 300 \mathrm{~km} \mathrm{~s}^{-1}$ compact groups. Filled and hatched histograms represent late-type galaxies in the $f_{\mathrm{E}}=0$ groups and those in the $f_{\mathrm{E}}>0$ groups, respectively. Right: The color distributions for late-type galaxies in the (d) $f_{\mathrm{E}}=0$, (e) $0<f_{\mathrm{E}}<0.5$, and (f) $0.5 \leq f_{\mathrm{E}}<1$ compact groups. Filled and hatched histograms represent late-type galaxies belonging to the $\sigma_{\mathrm{CG}}<100 \mathrm{~km} \mathrm{~s}^{-1}$ groups and the $\sigma_{\mathrm{CG}} \geq 100$ $\mathrm{km} \mathrm{s}^{-1}$ groups, respectively. We list $P_{\mathrm{KS}}$ and $P_{\mathrm{AD}}$ for the two histograms in each panel.
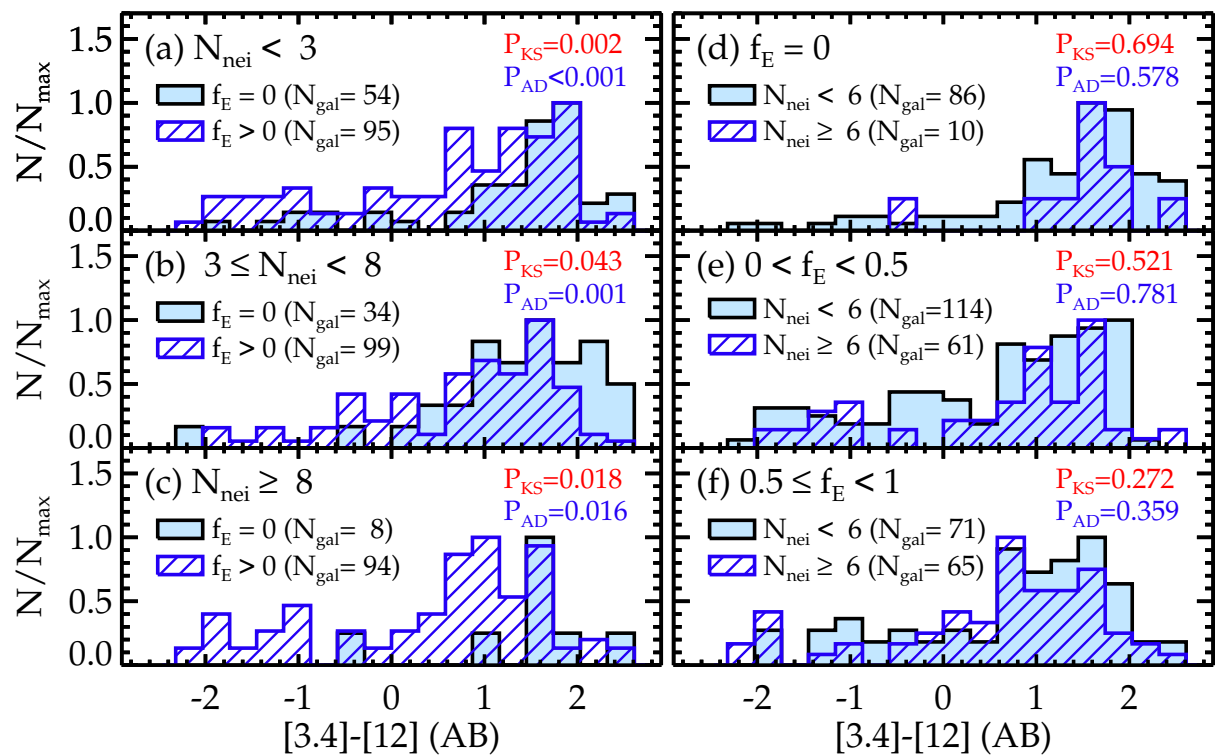

Fig. 11. - Left: MIR color distributions for late-type galaxies in the (a) $N_{\text {nei }}<3$, (b) $3<N_{\text {nei }}<8$, and (c) $N_{\text {nei }}>8$ compact groups. Filled and hatched histograms represent late-type galaxies in the $f_{\mathrm{E}}=0$ groups and those in the $f_{\mathrm{E}}>0$ groups, respectively. Right: The distributions of late-type galaxies in the (d) $f_{\mathrm{E}}=0$, (e) $0<f_{\mathrm{E}}<0.5$, and $0.5 \leq f_{\mathrm{E}}<1$ compact groups. Filled and hatched histograms represent late-type galaxies in the $N_{\text {nei }}<6$ compact groups and those in the $N_{\text {nei }} \geq 6$ compact groups, respectively. 


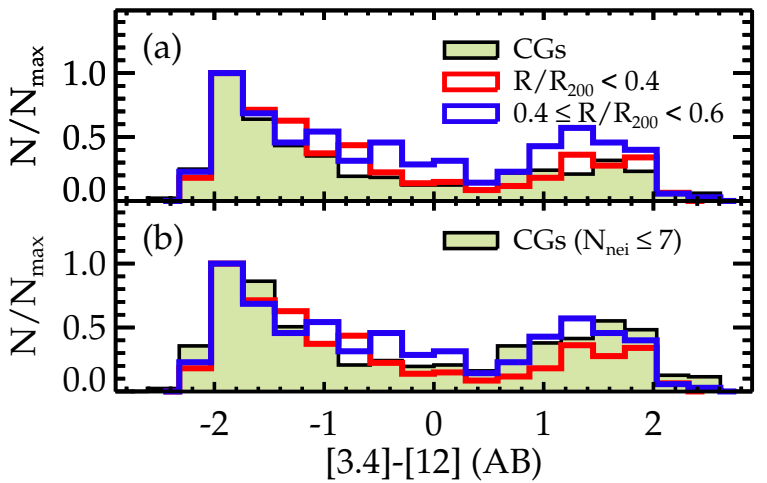

FIG. 12.- (a) Comparison of MIR color distributions for compact group galaxies (filled histograms) and cluster galaxies (open histograms). Red and blue histograms are for cluster galaxies with $R / R_{200}<0.4$ and $0.4 \leq R / R_{200}<0.4$, respectively. (b) We only plot galaxies belonging to $N_{\text {nei }} \leq 7$ compact groups.

in the $f_{\mathrm{E}}=0$ groups in all three $\sigma_{\mathrm{CG}}$ bins. The KS and $\mathrm{AD} \mathrm{k}$-sample tests reject the null hypothesis at a $\gtrsim 2.3 \sigma$ level in the three bins. On the other hand, in the right column of Figure 10 we compare MIR colors of late-type galaxies in the $\sigma_{\mathrm{CG}}<100 \mathrm{~km} \mathrm{~s}^{-1}$ groups with those of late-type galaxies in the $\sigma_{\mathrm{CG}} \geq 100 \mathrm{~km} \mathrm{~s}^{-1}$ groups in the three $f_{\mathrm{E}}$ bins. We find that there is no significant color difference between the two $\sigma_{\mathrm{CG}}$ samples in all three $f_{\mathrm{E}}$ bins. These results suggest that the MIR colors of latetype galaxies in compact groups are more sensitive to $f_{\mathrm{E}}$ than $\sigma_{\mathrm{CG}}$.

We conduct a similar analysis to examine whether the $f_{\mathrm{E}}$ effects on galaxy colors exist when $N_{\text {nei }}$ is fixed. Spearman's rank correlation coefficient for $f_{\mathrm{E}}$ and $N_{\text {nei }}$ is 0.39 , and the probability of obtaining the correlation by chance is $\ll 0.001 \%$, suggesting that there is a weak correlation between the two parameters. In the left column of Figure 11, we compare MIR colors of late-type galaxies in the $f_{\mathrm{E}}=0$ groups with those in the $f_{\mathrm{E}}>0$ groups in three $N_{\text {nei }}$ bins. In all three $N_{\text {nei }}$ bins, the MIR colors are bluer in the $f_{\mathrm{E}}>0$ groups than in the $f_{\mathrm{E}}=0$ groups. The AD k-sample test rejects the null hypothesis at the $\gtrsim 2.5 \sigma$ level, and the KS test also rejects the null hypothesis at the $>2 \sigma$ level. On the other hand, the right column shows that there is no significant difference in MIR colors between the late-type galaxy samples in the $N_{\text {nei }}<6$ groups and those in the $N_{\text {nei }} \geq 6$ groups. Figures 10] and 11]demonstrate that the MIR colors of late-type galaxies in compact groups change most significantly with $f_{\mathrm{E}}$ among the three environmental parameters, suggesting that the $f_{\mathrm{E}}$ is the most important parameter in determining the MIR colors of late-type galaxies in compact groups.

\section{DISCUSSION}

\subsection{Fast Galaxy Evolution in Compact Groups}

In Section 3. we find that the MIR colors of early-type galaxies are bluer in compact groups than in clusters and the field. This trend also persists when we use several subsamples with $N_{\text {nei }}, f_{\mathrm{E}}$, and $\sigma_{\mathrm{CG}}$. We also find that the late-type galaxies in the $f_{\mathrm{E}}>0$ (or $\sigma_{\mathrm{CG}} \geq 300 \mathrm{~km}$ $\mathrm{s}^{-1}$ ) compact groups have MIR colors bluer than those of cluster late-type galaxies. These results imply that stellar populations in early-type galaxies are on average older in compact groups than in clusters, and that star for- mation activity of late-type galaxies is suppressed more efficiently in $f_{\mathrm{E}}>0$ compact groups than in clusters. These results suggest that galaxy evolution in compact groups is faster than in the central $\left(R<0.5 R_{200}\right)$ regions of clusters.

So far, several studies have concluded that galaxy evolution is faster in compact groups than in the field through various analyses (e.g., Proctor et al. 2004; de la Rosa et al. 2007; Bitsakis et al. 2010, 2011, 2016; Tzanavaris et al. 2010; Walker et al. 2010, 2012; Coenda et al. 2012, 2015; Lenkić et al. 2016). Proctor et al. (2004) and Mendes de Oliveira et al. (2005) found that the properties of compact groups (i.e., stellar ages and the early-type galaxy fraction) are more similar to those of cluster galaxies than those of field galaxies. However, previous studies have not found evidence that galaxy evolution is faster in compact groups than in galaxy clusters.

Johnson et al. (2007) and Walker et al. (2010, 2012) showed that compact group galaxies show a strong bimodal Spitzer IRAC 3.6-8 $\mu$ m color distribution with an evident gap at green colors. This gap is not found in comparison samples of isolated galaxies, galaxy pairs, and the center of the Coma cluster. However, they found that the Coma infall region (i.e., $0.4-0.6 R_{200}$, Jenkins et al. 2007) shows the color distribution statistically similar to that of compact group galaxies. They interpreted these results as that the infall region and compact groups have a similarity in environment. To examine their results with our data, we select 495 galaxies at $R<0.4 R_{200}$ (representing the central regions of clusters) and 231 galaxies at $0.4 R_{200} \leq R<0.6 R_{200}$ (representing the infall regions) from the cluster galaxy sample. We compare the MIR color distributions for the two cluster galaxy subsamples with that for the compact group galaxy sample in Figure 12(a). The color distribution for the compact group galaxy sample is more similar to that for the $R<0.4 R_{200}$ cluster galaxy sample $\left(P_{\mathrm{KS}}=0.211\right.$ and $\left.P_{\mathrm{AD}}=0.102\right)$ than for the $0.4 R_{200} \leq R<0.6 R_{200}$ cluster galaxy sample $\left(P_{\mathrm{KS}}=0.001\right.$ and $\left.P_{\mathrm{AD}}=0.002\right)$. This result is not consistent with the results of Walker et al.

Walker et al. (2010) used a sample of 16 Hickson compact groups. Walker et al. (2012) used not only 21 Hickson compact groups but also 16 Redshift Survey Compact Groups (RSCG, Barton et al. 1996) identified using the friends-of-friends algorithm. However, only 5 of the 16 RSCGs are embedded in larger structures such as clusters. Thus, Walker et al.'s samples could also be dominated by compact groups located in less dense regions. We select 571 galaxies in the $N_{\text {nei }} \leq 7$ compact groups to mimic the Walker et al.'s sample, and show their MIR color distribution in Figure 12(b). The MIR color distribution of the 571 galaxies in the $N_{\text {nei }} \leq 7$ compact groups is not statistically different with the color distribution for the $0.4 R_{200} \leq R<0.6 R_{200}$ cluster galaxy sample $\left(P_{\mathrm{KS}}=0.444\right.$ and $\left.P_{\mathrm{AD}}=0.137\right)$, which is consistent with the results of Walker et al. Therefore, the different results between this study and Walker et al. are probably from the use of different methods in identifying compact groups with and without using isolation criterion (see also Figure 10 of Sohn et al. 2016).

One of the reasons why galaxy evolution is faster in compact groups than in clusters is likely because galaxy interactions are more frequent in compact groups than 


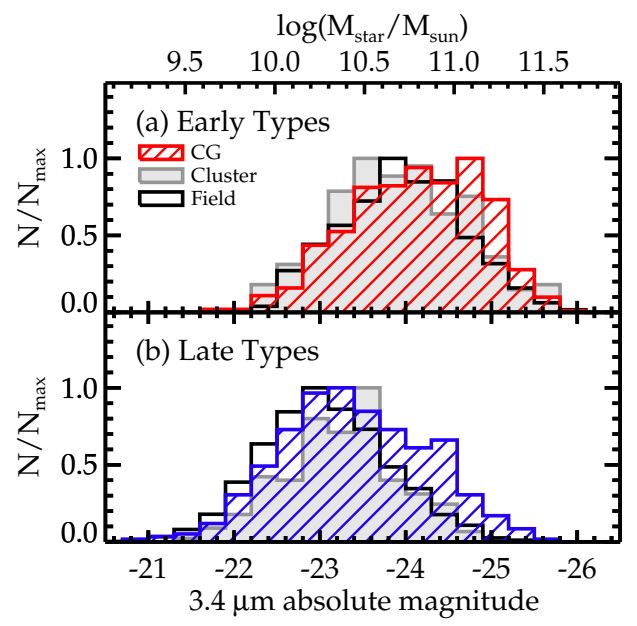

FIG. 13.- Comparison of $3.4 \mu \mathrm{m}$ absolute magnitude distributions between compact group (hatched histograms) and cluster galaxies (filled histograms). The top panel is for early-type galaxies, while the bottom panel is for late-type galaxies.

in clusters. The median velocity dispersion for our compact groups is $\sim 200 \mathrm{~km} \mathrm{~s}^{-1}$, which is much smaller than the median value for galaxy clusters, $\sim 800 \mathrm{~km} \mathrm{~s}^{-1}$ (Rines et al. 2013). Furthermore, the mean size of compact groups is $30.7 \pm 0.4 \mathrm{kpc}$, which is much smaller than the typical virial radius of galaxy clusters, $\sim 1-1.5 \mathrm{Mpc}$ (Park \& Hwang 2009; Rines et al. 2013). These characteristics make the interactions have a more significant effect in compact groups than in clusters.

In Figure 13, we compare the $3.4 \mu \mathrm{m}$ absolute magnitude $\left(M_{3.4}\right)$ distribution of early- and late-type galaxies in compact groups, clusters, and the field. Hwang et al. (2012b) showed that $M_{3.4}$ can be used as a proxy for stellar masses $\left(M_{\text {star }}\right)$ of galaxies. We confirm the correlation between the $M_{3.4}$ and $M_{\text {star }}$ using the compact group galaxies. The stellar masses in this study are based on $H_{0}=70 \mathrm{~km} \mathrm{~s}^{-1} \mathrm{Mpc}^{-1}$, and are calculated using the LePHARE ${ }^{8}$ spectral energy distribution fitting code (Arnouts et al. 1999; Ilbert et al. 2006) with the SDSS ugriz photometric data (see Section 2 of Sohn et al.|2016 for details). Table 2 lists $M_{\text {star }}$ and $M_{3.4}$ of compact group galaxies.

We find that among compact group early-type galaxies, the fraction of massive populations with $M_{3.4}<-24.5$ $\left(M_{\text {star }} \gtrsim 10^{11} M_{\text {sun }}\right)$ is $34.9 \% \pm 1.8 \%$, which is larger than the $24.6 \% \pm 2.1 \%$ for the cluster early-type galaxy sample and the $21.8 \% \pm 1.3 \%$ for field early-type galaxy sample. Similarly, among compact group late-type galaxies, the fraction of massive populations with $M_{3.4}<-23.5$ $\left(M_{\text {star }} \gtrsim 10^{10.5} M_{\text {sun }}\right)$ is $44.4 \% \pm 2.5 \%$, which is larger than the $32.9 \% \pm 3.2 \%$ for the cluster late-type galaxy sample and the $27.0 \% \pm 0.8 \%$ for the field late-type galaxy sample. This result suggests that compact groups are an ideal environment for efficient mass build-up of galaxies.

\subsection{Relation between Compact Group Members and Neighboring Galaxies}

In Section 3.3, as shown in Figure 6, we find that compact groups have larger $f_{\mathrm{E}}$ and bluer MIR colors as their

\footnotetext{
${ }^{7} \log \left(M_{\text {star }} / M_{\text {sun }}\right)=(-0.48 \pm 0.01) \times M_{3.4}-(0.76 \pm 0.20)$ with $\mathrm{rms}=0.27$.

${ }^{8}$ http://www.cfht.hawaii.edu/ arnouts/LEPHARE/lephare.html
}

surrounding environments are denser (larger $N_{\text {nei }}$ ). To further investigate the effects of the surrounding environments on the physical properties of compact group member galaxies, we compare the properties of neighboring galaxies around each compact group with those of the compact group member galaxies. We calculate $f_{\mathrm{E}}$, mean $u-r(\overline{u-r})$, and mean [3.4] - [12] $(\overline{[3.4]-[12]})$ for neighboring galaxies in the comoving cylinder of each compact group. Figure $\left[14\right.$ shows $f_{\mathrm{E}}, \overline{u-r}$, and $\overline{[3.4]-[12]}$ for neighboring galaxies as a function of $N_{\text {nei }}$. Triangles indicate the mean values of the three parameters in $N_{\text {nei }}$ bins. As $N_{\text {nei }}$ increases, neighboring galaxies have larger $f_{\mathrm{E}}$, redder optical colors, and bluer MIR colors. For comparison, we compute $f_{\mathrm{E}}, \overline{u-r}$, and $\overline{[3.4]-[12]}$ for member galaxies of each compact group. Squares represent the mean values for the three parameters of compact group member galaxies.

We find that the two mean curves show a similar pattern in all three panels. The $N_{\text {nei-dependence of }}$ $f_{\mathrm{E}}, \overline{u-r}$, and $\overline{[3.4]-[12]}$ for neighboring galaxies is consistent with the morphology-density or the SFRdensity relation (e.g., Dressler 1980; Lewis et al. 2002; Park \& Hwang 2009). However, it is interesting that the similar trends are also seen for compact group member galaxies. Since compact groups are very small in size (the mean size is $30.7 \pm 0.4 \mathrm{kpc}$ ), their internal galaxy number densities are much higher than the surrounding densities. The mean internal density of compact groups, measured by the number of members divided by the sizes of the groups, is $1034 \pm 18 \mathrm{Mpc}^{-2}$, which is much higher than the mean surrounding density, 2.54 $\pm 0.10 \mathrm{Mpc}^{-2}$, from $N_{\text {nei }}$. Moreover, the internal galaxy number densities of compact groups do not depend on the surrounding galaxy number densities. Thus, the $N_{\text {nei-dependence of }}$ the properties of compact group member galaxies can not be explained by the morphology-density or SFR-density relations alone.

The similar $N_{\text {nei-dependence for compact group mem- }}$ ber galaxies and their neighboring galaxies suggests that the properties of compact group galaxies are related to those of their neighboring galaxies. If the physical properties of compact group galaxies are independent of their neighboring galaxies, the two mean curves would show different behaviors. A plausible scenario would be that the neighboring galaxies are sources of member galaxies in compact groups. This supports the idea of Diaferio et al. (1994) that compact groups replenish their members from surrounding environments, so that they do not disappear by mergers within a few Gyrs. Previous studies have supported this replenishment model by showing that many $(>50 \%)$ compact groups are associated with larger-scale galaxy structures that may supply new members of compact groups. On the other hand, our finding is more direct observational evidence that the replenishment occurs regardless of the surrounding density of compact groups.

An important point is that compact groups are not simply aggregates of captured neighboring galaxies. Figure 14 shows that the two mean curves do not overlap, but differ systematically. This result suggests that there are additional environmental effects playing a critical role in morphology transformation and star formation quenching for compact group galaxies, which prob- 

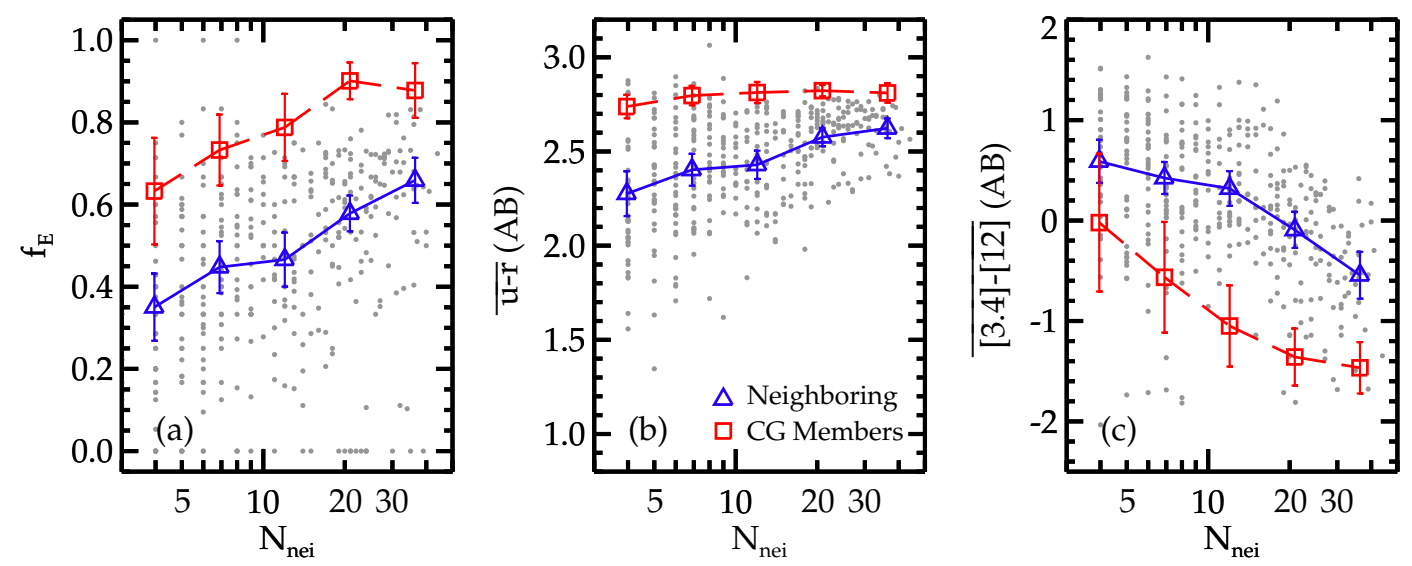

FIG. 14.- $N_{\text {nei }}$ dependence of (a) $f_{\mathrm{E}}$, (b) $\overline{u-r}$, and (c) $\overline{[3.4]-[12]}$ for neighboring galaxies within the comoving cylinder around the compact groups, and their mean values (triangles) in $N_{\text {nei }}$ bins. For comparison, we also plot the mean values of the three parameters for compact group member galaxies in $N_{\text {nei }}$ bins (squares). Error bars represent $3 \sigma$ deviation in a given $N_{\text {nei }}$ bin.

ably results from frequent interactions among member galaxies. This result also suggests that compact groups are the most suitable environment for the pre-processing (Zabludoff \& Mulchaev 1998).

\subsection{Hydrodynamic Interactions in Compact Groups}

We find that the MIR colors of late-type galaxies are bluer in the $f_{\mathrm{E}}>0$ compact groups than in the $f_{\mathrm{E}}=0$ compact groups (Figure 8). This $f_{\mathrm{E}}$ dependence is still found when $N_{\text {nei }}$ or $\sigma_{\mathrm{CG}}$ is fixed (see Figures 10 and 11), indicating that among the three environmental parameters, $f_{\mathrm{E}}$ could be the most important parameter in determining the MIR colors of late-type member galaxies. The $[3.4]-[12]$ colors of late-type galaxies are well correlated with specific SFRs (Donoso et al. 2012; Hwang et al. 2012b). Thus, this finding suggests that star formation activity of late-type galaxies is suppressed more efficiently in the $f_{\mathrm{E}}>0$ compact groups than in the $f_{\mathrm{E}}=0$ compact groups. Moreover, the suppression of star formation activity is stronger in the $f_{\mathrm{E}}>0$ compact groups than in the cluster central $\left(R<0.5 R_{200}\right)$ regions (Figure 8).

Bitsakis et al. (2010, 2011) showed that late-type galaxies in the $f_{\mathrm{E}}>0.25$ compact groups tend to have smaller specific SFRs than those in the $f_{\mathrm{E}}<0.25$ compact groups. Their result seems similar to our result. To verify their result with our sample, we divide our compact group sample into the $f_{\mathrm{E}}=0,0<f_{\mathrm{E}} \leq 0.25$, and $f_{\mathrm{E}}>0.25$ compact groups. The large values $(>0.85)$ of $P_{\mathrm{KS}}$ and $P_{\mathrm{AD}}$ cannot reject the null hypothesis for two MIR color distributions for late-type galaxies in the $0<f_{\mathrm{E}} \leq 0.25$ compact groups and for late-type galaxies in the $f_{\mathrm{E}}>0.25$ compact groups. However, the $P_{\mathrm{KS}}=0.056$ and $P_{\mathrm{AD}}=0.008$ reject the null hypothesis for two MIR color distributions for late-type galaxies in the $f_{\mathrm{E}}=0$ compact groups and for late-type galaxies in the $0<f_{\mathrm{E}} \leq 0.25$ compact groups at a significance $\gtrsim 2 \sigma$. These suggest that the $f_{\mathrm{E}}=0$ and $0<f_{\mathrm{E}} \leq 0.25$ compact groups in our sample have statistically different MIR color distributions for their late-type member galaxies, which supports again our finding that MIR colors of late-type galaxies are bluer in the $f_{\mathrm{E}}>0$ compact groups than in the $f_{\mathrm{E}}=0$ compact groups. The different $f_{\mathrm{E}}$ criterion between this study and Bitsakis et al. probably results from different compact group samples.
An important point here is that the star formation activity of late-type galaxies in compact groups is suppressed when the groups contain early-type members. This suppression of star formation activity cannot be explained by gravitational interactions among member galaxies alone. Park et al. (2008) and Park \& Choi (2009) found that galaxy properties strongly depend on the distance and morphology of the nearest neighbor galaxy, using spectroscopic samples drawn from the SDSS data. They showed that when a galaxy is located within the virial radius of its nearest neighbor galaxy, the galaxy tends to have a morphological type similar to that of the neighbor galaxy. However, this phenomenon does not manifest if the distance to the neighbor is greater than the virial radius of the neighbor. They suggested that the phenomenon is due to hydrodynamic interactions, and that the effects of hydrodynamic interactions are significant to change the morphology and star formation activity of galaxies when the galaxies are located within the virial radii of their neighbor galaxies. The sizes of compact groups (the mean value is $30.7 \mathrm{kpc}$ ) are significantly smaller than virial radii of member galaxies (i.e., $430 \mathrm{kpc}$ and $340 \mathrm{kpc}$ for early- and late-type galaxies with $M_{r}=-20.77$, Park \& Hwang 2009). This implies that compact group member galaxies are already located within the virial radius of each other, and that they interact hydrodynamically each other.

Park \& Hwang (2009) focused on the case of galaxies located within the virial radii of massive galaxy clusters, and found that even in cluster environments the hydrodynamic interactions with early-type neighbor galaxies are the main drivers of star formation quenching of late-type galaxies. They also found that the effects of the cluster hot gas on the star formation quenching of galaxies at $R>0.1 R_{200}$ is insignificant compared to the effects of hydrodynamic interactions with neighbor galaxies. Unlike cluster environments, hot gas of compact groups is not in a hydrostatic equilibrium state, and it is likely to be associated with the individual galaxies or brightest galaxies (Desiardins et al. 2013, 2014), suggesting that hydrodynamic effects from hot gas are insignificant in compact groups. Therefore, we conclude that the suppressed star formation activity of late-type galaxies in the $f_{\mathrm{E}}>0$ compact groups likely results from hydrody- 
namic interactions with early-type member galaxies.

\section{SUMMARY AND CONCLUSIONS}

We study the MIR properties of galaxies in compact groups and their environmental dependence using a volume-limited sample of 670 compact groups and their 2175 member galaxies with $M_{r}<-19.77$ and $0.01<z<0.0741$, drawn from the catalog of Sohn et al. (2016). This catalog was derived from nearly complete redshift survey data, and was constructed by applying a friends-of-friends method without applying Hickson's isolation criterion, which make the catalog include nearby compact groups and embedded compact groups in highdensity regions that were often excluded from previous catalogs. Using this unbiased compact group sample, we perform a study comparing the physical properties of galaxies in compact groups with those of galaxies in other environments such as galaxy clusters and the field. We use three environmental parameters including $N_{\text {nei }}$, $f_{\mathrm{E}}$, and $\sigma_{\mathrm{CG}}$ to represent the internal and external environment of the compact groups. Our key findings of this study are summarized as follows.

1. The MIR colors of compact group early-type galaxies are on average bluer than those of cluster $(R<$ $\left.0.5 R_{200}\right)$ early-type galaxies regardless of $N_{\text {nei }}, f_{\mathrm{E}}$, and $\sigma$. This suggests that early-type galaxies in compact groups are on average older than those of cluster galaxies.

2. The MIR colors of the late-type galaxies in the $f_{\mathrm{E}}>0$ compact groups tend to be bluer than those of cluster late-type galaxies. These suggests that the star formation activity of late-type galaxies is suppressed more efficiently in $f_{\mathrm{E}}>0$ compact groups than in clusters.

3. As $N_{\text {nei }}$ increases, compact groups have larger $f_{\mathrm{E}}$, redder optical colors, and bluer MIR colors. These trends are also seen for neighboring galaxies around compact groups. Considering the extremely high galaxy number densities in compact group environments, this similar $N_{\text {nei-dependence for com- }}$ pact group member galaxies and their neighboring galaxies is not well explained by the morphology- and SFR-density relations. This result can be explained by the scenario that neighboring galaxies are sources of member galaxies in compact groups, supporting the replenishment model suggested by Diaferio et al. (1994).

4. At a given $N_{\text {nei }}$, compact group members always have on average larger $f_{\mathrm{E}}$ and bluer MIR colors than neighboring galaxies. This suggests that compact groups are not simply aggregates of captured neighboring galaxies, and that compact group environments play a critical role in accelerating morphology transformation and star formation quenching for the member galaxies.

5. In the $f_{\mathrm{E}}=0$ compact groups, the MIR colors of late-type galaxies are on average redder than those of cluster late-type galaxies. However, in the $f_{\mathrm{E}}>0$ compact groups, the MIR colors of late-type galaxies are on average bluer than those in cluster late-type galaxies. This indicates that star formation quenching occurs more effectively in compact groups than in clusters when compact groups have early-type members. This suppressed star formation activity of late-type galaxies likely results from hydrodynamic interactions with early-type member galaxies.

Our results suggest that galaxy evolution is faster in compact groups than in other environments, and that compact groups are likely to be the best place for the pre-processing (Zabludoff \& Mulchaev 1998).

We thank the anonymous referee for useful and constructive comments that helped us to improve the manuscript. We also thank Brian S. Cho for helping the English in the manuscript improved. G.H.L. acknowledges the support by the National Research Foundation of Korea (NRF) Grant funded by the Korean Government (NRF-2012-Fostering Core Leaders of the Future Basic Science Program). M.G.L was supported by the NRF grant funded by the Korea Government (MEST) (No. 2012R1A4A1028713).

\section{REFERENCES}

Abazajian, K. N., Adelman-McCarthy, J. K., Agüeros, M. A., et al. 2009, ApJS, 182, 543

Adelman-McCarthy, J. K., Agüeros, M. A., Allam, S. S., et al. 2008, ApJS, 175, 297-313

Alam, S., Albareti, F. D., Allende Prieto, C., et al. 2015, ApJS, 219, 12

Alatalo, K., Cales, S. L., Appleton, P. N., et al. 2014, ApJ, 794 L13

Amram, P., Mendes de Oliveira, C., Plana, H., Balkowski, C., \& Hernandez, O. 2007, A\&A, 471, 753

Appleton, P. N., Xu, K. C., Reach, W., et al. 2006, ApJ, 639, L51

Arnouts, S., Cristiani, S., Moscardini, L., et al. 1999, MNRAS, 310,540

Barnes, J. 1985, MNRAS, 215, 517

Barnes, J. E. 1989, Nature, 338, 123

Barton, E., Geller, M., Ramella, M., Marzke, R. O., \& da Costa, L. N. 1996, AJ, 112, 871

Bitsakis, T., Charmandaris, V., Le Floc'h, E., et al. 2010, A\&A 517, A 75

Bitsakis, T., Charmandaris, V., da Cunha, E., et al. 2011, A\&A, 533, A 142

Bitsakis, T., Dultzin, D., Ciesla, L., et al. 2015, MNRAS, 450, 3114
Bitsakis, T., Dultzin, D., Ciesla, L., et al. 2016, MNRAS, 459, 957 Blanton, M. R., \& Roweis, S. 2007, AJ, 133, 734

Choi, Y.-Y., Han, D.-H., \& Kim, S. S. 2010, Journal of Korean Astronomical Society, 43, 191

Cluver, M. E., Appleton, P. N., Boulanger, F., et al. 2010, ApJ, 710,248

Cluver, M. E., Appleton, P. N., Ogle, P., et al. 2013, ApJ, 765, 93

Coenda, V., Muriel, H., \& Martínez, H. J. 2012, A\&A, 543, A119

Coenda, V., Muriel, H., \& Martínez, H. J. 2015, A\&A, 573, A96

Coziol, R., \& Plauchu-Frayn, I. 2007, AJ, 133, 2630

Coziol, R., Ribeiro, A. L. B., de Carvalho, R. R., \& Capelato, H. V. 1998, ApJ, 493, 563

Coziol, R., de Carvalho, R. R., Capelato, H. V., \& Ribeiro,

A. L. B. 1998, ApJ, 506, 545

de Carvalho, R. R., Ribeiro, A. L. B., \& Zepf, S. E. 1994, ApJS, 93,47

de la Rosa, I. G., de Carvalho, R. R., Vazdekis, A., \& Barbuy, B. 2007, AJ, 133, 330

Desjardins, T. D., Gallagher, S. C., Tzanavaris, P., et al. 2013, ApJ, 763, 121

Desjardins, T. D., Gallagher, S. C., Hornschemeier, A. E., et al. 2014, ApJ, 790, 132

Diaferio, A., Geller, M. J., \& Ramella, M. 1994, AJ, 107, 868 
Díaz-Giménez, E., \& Zandivarez, A. 2015, A\&A, 578, A61

Donoso, E., Yan, L., Tsai, C., et al. 2012, ApJ, 748, 80

Dressler, A. 1980, ApJ, 236, 351

Falco, E. E., Kurtz, M. J., Geller, M. J., et al. 1999, PASP, 111 438

Gallagher, S. C., Durrell, P. R., Elmegreen, D. M., et al. 2010 , AJ, 139,545

Hickson, P. 1982, ApJ, 255, 382

Hill, J. M., \& Oegerle, W. R. 1993, AJ, 106, 831

Hill, J. M., \& Oegerle, W. R. 1998, AJ, 116, 1529

Hwang, H. S., Elbaz, D., Lee, J. C., et al. 2010, A\&A, 522, A33

Hwang, H. S., Park, C., Elbaz, D., \& Choi, Y.-Y. 2012a, A\&A, $538, \mathrm{~A} 15$

Hwang, H. S., Geller, M. J., Kurtz, M. J., Dell'Antonio, I. P., \& Fabricant, D. G. 2012b, ApJ, 758, 25

Ilbert, O., Arnouts, S., McCracken, H. J., et al. 2006, A\&A, 457, 841

Jenkins, L. P., Hornschemeier, A. E., Mobasher, B., Alexander, D. M., \& Bauer, F. E. 2007, ApJ, 666, 846

Johnson, K. E., Hibbard, J. E., Gallagher, S. C., et al. 2007, AJ, 134,1522

Kewley, L. J., Groves, B., Kauffmann, G., \& Heckman, T. 2006, MNRAS, 372,961

Ko, J., Im, M., Lee, H. M., et al. 2009, ApJ, 695, L198

Ko, J., Hwang, H. S., Lee, J. C., \& Sohn, Y.-J. 2013, Ap.J, 767, 90

Ko, J., Chung, H., Hwang, H. S., \& Lee, J. C. 2016, ApJ, 820, 132

Konstantopoulos, I. S., Gallagher, S. C., Fedotov, K., et al. 2012, ApJ, 745, 30

Kroupa, P. 2015, Canadian Journal of Physics, 93, 169

Lee, G.-H., Hwang, H. S., Lee, M. G., et al. 2015, ApJ, 800, 80

Lenkić, L., Tzanavaris, P., Gallagher, S. C., et al. 2016, MNRAS, 459,2948

Lewis, I., Balogh, M., De Propris, R., et al. 2002, MNRAS, 334, 673

Mamon, G. A. 1987, ApJ, 321, 622

Mateos, S., Alonso-Herrero, A., Carrera, F. J., et al. 2012, MNRAS, 426, 3271

Martínez, M. A., Del Olmo, A., Coziol, R., \& Perea, J. 2010, AJ, 139, 1199

McConnachie, A. W., Patton, D. R., Ellison, S. L., \& Simard, L. 2009, MNRAS, 395, 255

Mendel, J. T., Ellison, S. L., Simard, L., Patton, D. R., \& McConnachie, A. W. 2011, MNRAS, 418, 1409

Mendes de Oliveira, C., Coelho, P., González, J. J., \& Barbuy, B. 2005, AJ, 130, 55

Muratov, A. L., \& Gnedin, O. Y. 2010, ApJ, 718, 1266

Park, C., \& Choi, Y.-Y. 2005, ApJ, 635, L29

Park, C., \& Choi, Y.-Y. 2009, ApJ, 691, 1828

Park, C., \& Hwang, H. S. 2009, ApJ, 699, 1595

Park, C., Gott, J. R., III, \& Choi, Y.-Y. 2008, ApJ, 674, 784-796

Plauchu-Frayn, I., \& Coziol, R. 2010, AJ, 140, 612
Plauchu-Frayn, I., Del Olmo, A., Coziol, R., \& Torres-Papaqui, J. P. 2012, A\&A, 546, A48

Piovan, L., Tantalo, R., \& Chiosi, C. 2003, A\&A, 408, 559

Pompei, E., \& Iovino, A. 2012, A\&A, 539, A106

Proctor, R. N., Forbes, D. A., Hau, G. K. T., et al. 2004,

MNRAS, 349, 1381

Ramella, M., Diaferio, A., Geller, M. J., \& Huchra, J. P. 1994 AJ, 107, 1623

Ribeiro, A. L. B., de Carvalho, R. R., Capelato, H. V., \& Zepf, S. E. 1998, ApJ, 497, 72

Rines, K., Geller, M. J., Diaferio, A., \& Kurtz, M. J. 2013, ApJ, 767, 15

Rodrigue, M., Schultz, A., Thompson, J., et al. 1995, AJ, 109, 2362

Rood, H. J., \& Struble, M. F. 1994, PASP, 106, 413

Rubin, V. C., Hunter, D. A., \& Ford, W. K., Jr. 1990, ApJ, 365 86

Schawinski, K., Urry, C. M., Simmons, B. D., et al. 2014,

MNRAS, 440, 889

Schlegel, D. J., Finkbeiner, D. P., \& Davis, M. 1998, ApJ, 500, 525

Slinglend, K., Batuski, D., Miller, C., et al. 1998, ApJS, 115, 1

Sohn, J., Hwang, H. S., Lee, M. G., Lee, G.-H., \& Lee, J. C. 2013, ApJ, 771, 106

Sohn, J., Hwang, H. S., Geller, M. J., et al. 2015, Journal of Korean Astronomical Society, 48, 381

Sohn, J., Geller, M. J., Hwang, H. S., Zahid, H. J., \& Lee, M. G. 2016, ApJS, 225, 23

Tegmark, M., Blanton, M. R., Strauss, M. A., et al. 2004, ApJ, 606,702

Tzanavaris, P., Hornschemeier, A. E., Gallagher, S. C., et al. 2010, ApJ, 716, 556

Tzanavaris, P., Gallagher, S. C., Hornschemeier, A. E., et al. 2014, ApJS, 212, 9

Vogt, F. P. A., Dopita, M. A., Borthakur, S., et al. 2015, MNRAS, 450, 2593

Walker, L. M., Johnson, K. E., Gallagher, S. C., et al. 2010, AJ, 140,1254

Walker, L. M., Johnson, K. E., Gallagher, S. C., et al. 2012, AJ, 143,69

Walker, L. M., Butterfield, N., Johnson, K., et al. 2013, ApJ, 775, 129

Wegner, G., Colless, M., Baggley, G., et al. 1996, ApJS, 106, 1

Wegner, G., Colless, M., Saglia, R. P., et al. 1999, MNRAS, 305, 259

Wright, E. L., Eisenhardt, P. R. M., Mainzer, A. K., et al. 2010, AJ, 140,1868

Zabludoff, A. I., \& Mulchaey, J. S. 1998, ApJ, 496, 39

Zucker, C., Walker, L. M., Johnson, K., et al. 2016, ApJ, 821, 113 